\title{
Heparan Sulfate-dependent RAGE oligomerization is indispensable for pathophysiological functions of RAGE
}

\author{
Miaomiao $\mathrm{Li}^{1}$, Chih Yean Ong ${ }^{1}$, Christophe J Langouët-Astrié ${ }^{2}$, Lisi Tan ${ }^{1,3}$, Ashwni Verma ${ }^{4}$, Yimu \\ Yang $^{2}$, Xiaoxiao Zhang ${ }^{1}$, Dhaval K. Shah ${ }^{4}$, Eric P. Schmidt ${ }^{2}$ and Ding $\mathrm{Xu}^{1 *}$ \\ ${ }^{1}$ Department of Oral Biology, School of Dental Medicine, University at Buffalo, the State \\ University of New York. \\ ${ }^{2}$ Division of Pulmonary Sciences and Critical Care Medicine, Department of Medicine, \\ University of Colorado Anschutz Medical Campus, Aurora, Colorado. \\ ${ }^{3}$ Department of Periodontics, School of Stomatology, China Medical University, Shenyang, \\ China. \\ ${ }^{4}$ Department of Pharmaceutical Sciences, School of Pharmacy and Pharmaceutical Sciences, \\ University at Buffalo, the State University of New York.
}

* To whom correspondence and proofs should be sent: Department of Oral Biology, School of Dental Medicine, University at Buffalo, SUNY, Buffalo, NY 14214. Phone: 716-829-3509, E-mail: dingxu@buffalo.edu

\section{Keywords}

Heparan sulfate, RAGE, inflammation, osteoclast, oligomerization 


\begin{abstract}
RAGE, a druggable inflammatory receptor, is known to function as an oligomer but the exact oligomerization mechanism remains poorly understood. Previously we have shown that heparan sulfate (HS) plays an active role in RAGE oligomerization. To understand the physiological significance of HS-induced RAGE oligomerization in vivo, we generated RAGE knock-in mice $\left(\right.$ Rage $\left.^{\mathrm{AHA} / \mathrm{AHA}}\right)$ by introducing point mutations to specifically disrupt HS-RAGE interaction. The RAGE mutant demonstrated normal ligand-binding but impaired capacity of HS-binding and oligomerization. Remarkably, Rage $\mathrm{AHA}^{\mathrm{AHA}}$ mice phenocopied Rage $\mathrm{e}^{-/}$mice in two different pathophysiological processes, namely bone remodeling and neutrophil-mediated liver injury, which demonstrates that HS-induced RAGE oligomerization is essential for RAGE signaling. Our findings suggest that it should be possible to block RAGE signaling by inhibiting HS-RAGE interaction. To test this, we generated a monoclonal antibody that targets the HS-binding site of RAGE. This antibody blocks RAGE signaling in vitro and in vivo, recapitulating the phenotype of Rage $\mathrm{AHA}^{\mathrm{AHAHA}}$ mice. By inhibiting HS-RAGE interaction genetically and pharmacologically, our work validated an alternative strategy to antagonize RAGE. Finally, we have performed RNAseq analysis of neutrophils and lungs and found that while $R a g e^{-/-}$mice had a broad alteration of transcriptome in both tissues compared to wild-type mice, the changes of transcriptome in

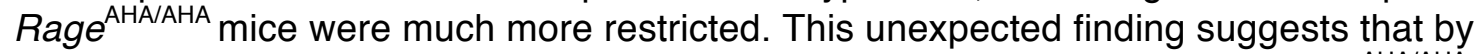
preserving the expression of RAGE protein (in a dominant-negative form), Rage AHA/AHA $_{\text {mouse }}$ might represent a cleaner genetic model to study physiological roles of RAGE in vivo compared to Rage $^{-/-}$mice.
\end{abstract}

\title{
INTRODUCTION
}

Receptor for Advanced Glycation End-Products (RAGE) is a transmembrane protein belonging to the immunoglobulin (Ig) superfamily of receptors. RAGE has attracted tremendous interest in the last two decades given its involvement in many disease conditions including diabetes, Alzheimer's, atherosclerosis, cancer, and many other inflammatory disorders $(1,2)$. RAGE binds a wide array of ligands including advanced glycation end products, amyloid $\beta$ peptide, S100 family proteins, and high mobility group box 1 (HMGB1). In most pathological conditions, diseased tissues show a dramatically enhanced expression and activation of RAGE. Ablation of RAGE signaling in these conditions, either by neutralizing RAGE ligands or by using RAGE knockout mice, has resulted in greatly improved outcome in murine models, which strongly suggests that RAGE can be pursued as a promising therapeutic target (3-8).

While many pathological roles of RAGE have been well established, the homeostatic role of RAGE remained a mystery until 2006, when it was found that RAGE plays an essential role in regulating osteoclast differentiation and maturation (9). While unchallenged RAGE null mice display normal homeostasis in all other aspects, they demonstrated increased bone mineral density. Mechanistically, it was revealed that HMGB1 secreted by preosteoclasts activates RAGE in an autocrine fashion, and HMGB1-RAGE interaction plays a critical role in establishing the sealing zone, a structural feature of osteoclasts essential for bone resorption (10).

Recently, HMGB1-RAGE signaling has been shown to also play a key role in drug-induced liver injury (6). Following acetaminophen (APAP) overdose, hepatocytes undergoing necrosis release HMGB1 as a danger signal to promote tissue repair. However, when extensive tissue necrosis occurs, the exaggerated inflammation, mediated by HMGB1-driven, RAGE-dependent neutrophil infiltration, often leads to secondary liver damage with worsened disease outcome. In a mouse of APAP-induced liver injury, we and others demonstrated that Rage ${ }^{-/}$mice were greatly protected from liver damage chiefly due to suppression of neutrophil infiltration $(3,6)$. 
Like many cell surface receptors, RAGE signaling depends on receptor oligomerization (11-13). However, the exact mechanism by which RAGE oligomerizes remains controversial (14). Historically, RAGE has been hypothesized to undergo ligand-dependent oligomerization (15). We have proposed an alternative hypothesis: that prior to ligand binding, RAGE preassembles into an oligomeric complex by interacting with heparan sulfate (HS), a negatively charged polysaccharide widely-expressed at the cell surface. This alternative hypothesis is supported by in depth structural analyses, where we showed by both crystal and solution structures that HS oligosaccharides induce RAGE to form a stable hexamer in the absence of ligand (16). Furthermore, in the same study we showed that in endothelial cells, activation of RAGE signaling by all tested RAGE ligands, including HMGB1, S100A8/A9, S100A12, S100B and AGE, depended upon the presence of $\mathrm{HS}$ at the cell surface. Based upon these findings, we propose that HS functions as a main driver of RAGE oligomerization, assembling RAGE into an oligomeric complex at cell surface without activating it. Only this oligomeric complex can serve as a functional receptor, which can then be activated by RAGE ligands to initiate signaling. As virtually all mammalian cells express HS, HS-dependent RAGE oligomerization is likely a universal requirement for RAGE signaling regardless of cell types and ligands involved.

To investigate the physiological significance of HS-RAGE interaction and HS-dependent RAGE oligomerization, we generated a novel RAGE knock-in (Rage ${ }^{\mathrm{AHA} / \mathrm{AHA}}$ ) mouse model by introducing point mutations into residues that are involved in HS binding. Remarkably, when bone morphometric indexes and susceptibility to APAP-induced liver injury were examined, we found that the Rage $\mathrm{AHA}^{\mathrm{AHA} A}$ mice phenocopies $R_{a g e^{-/}}$mice precisely. Our findings strongly suggest that HS-RAGE interaction is required for normal function of RAGE in multiple cellular systems and that targeting HS-RAGE interaction could represent a new opportunity to curb RAGE activation. By developing a monoclonal antibody $(\mathrm{mAb})$ that specifically targets part of the HS-binding site of RAGE, we demonstrated that this novel targeting strategy was indeed effective in inhibiting osteoclastogenesis and APAP-induced liver injury. Finally, we discovered that compared to Rage ${ }^{1-}$ mice, alterations in global transcriptome was much more restricted in Rage ${ }^{\mathrm{AHA} / \mathrm{AHA}}$ mice, which suggest that Rage ${ }^{\mathrm{AHA} / \mathrm{AHA}}$ mice might be a better murine model to study RAGE-dependent biological processes.

\section{RESULTS}

\section{Characterization of RAGE-AHA mutant and generation of Rage ${ }^{\mathrm{AHA} / \mathrm{AHA}}$ knock in mice}

RAGE is a multidomain protein consisting of three extracellular immunoglobulin domains (V-C1C2, also known as sRAGE), a single transmembrane domain, and a short cytoplasmic tail (17, 18). We previously reported that heparan sulfate can induce RAGE to form a hexamer (trimer of dimers) with 1:2 stoichiometry, and that the interaction involves five basic residues in the $\mathrm{V}$ domain and two residues (R216 and $R 218$ ) in the $C 1$ domain (Fig. 1A) (16). Here we prepared a triple mutant of mouse RAGE VC1 domain bearing R216A, R217H and R218A mutations (mVC1-AHA). As expected, the binding capacity of $\mathrm{mVC1}$-AHA to the heparin Sepharose column was reduced significantly compared with wildtype mouse RAGE VC1 domain (WT-mVC1) (Fig. 1B). Furthermore, mVC1-AHA was unable to form stable hexamer in the presence of HS dodecasaccharides (Fig. 1C). Importantly, mVC1-AHA maintains WT-like binding affinity to RAGE ligand HMGB1 (apparent binding affinity $9.9 \mathrm{nM}$ versus $11.7 \mathrm{nM}$, Fig. 1D), which is expected because binding of RAGE ligands predominantly occurs at the $V$ domain. To generate a murine model to specifically disrupt HS-RAGE interaction, we introduced R216A-R217H-R218A triple mutations into the ager (synonymous with rage) locus by CRISPR-Cas9 mediated homologous recombination (Fig. 1E). Rage $\mathrm{AHA} / \mathrm{AHA}$ mice were born with normal litter size and grew normally without gross morphological abnormalities. The RAGE expression level in Rage AHA/AHA $_{\text {mice lungs }}$ 
was similar to WT mice, which suggested that the mutation did not negatively affect the expression of RAGE (Fig. 1F).

\section{$\boldsymbol{R a g e}^{\mathrm{AHA} / \mathrm{AHA}}$ and $\boldsymbol{R a g e}^{\mathrm{AHA} / \mathrm{+}}$ mice develop an osteopetrotic phenotype}

As RAGE was reported to be associated with osteoclast maturation, we first examined the bone

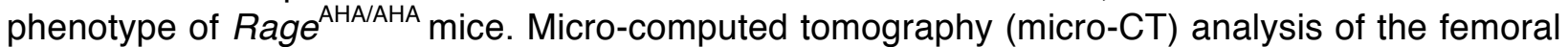
trabecular bone morphology and microarchitecture showed that 10 -week old male Rage AHA/AHA $^{-1}$ mice exhibited increased trabecular bone mass (Fig. 2A) and displayed a $49 \%$ increase in bone volume over tissue volume (BV/TV) (Fig. 2B), 17\% increase in trabecular thickness (Tb.Th) and $15 \%$ decrease in trabecular separation (Tb.Sp) (Fig. S1). In addition, these Rage ${ }^{\mathrm{AHA} / \mathrm{AHA}}$ mice also displayed a significant increase in the cortical bone thickness (from 0.182 to $0.205 \mathrm{~mm}$ ) (Fig. 2C). Similar phenotype was also observed in 10-week old female Rage ${ }^{\text {AHA/AHA }}$ mice, which displayed $70 \%$ increase in bone volume compared to WT mice (Fig. S2). Notably, all these parameters are highly similar to those of 10-week old Rage $^{-/-}$mice (Fig. 1A-C and Fig. S1-S2). Interestingly, when they were 4-weeks old, the Rage $e^{\mathrm{AHA} / \mathrm{AHA}}$ mice had normal bone mass, while Rage ${ }^{-/-}$mice had slightly lower bone mass compared to WT mice. By 4-months old, while the bone mass of Rage $e^{\mathrm{AHA} / \mathrm{AHA}}$ mice was $26 \%$ higher than WT mice, the bone mass of $R a g e^{-/-}$mice were significantly higher ( $75 \%$ over WT). Taken together, these data indicate that the interaction between HS and RAGE, and HS induced oligomer formation, are indispensable for RAGE to function in bone remodeling.

To understand the potential contribution of haploinsufficiency and RAGE gene dosage in bone phenotype, we examined both strains of heterozygous mice. To our surprise, male Rage $\mathrm{AHA} /+^{\mathrm{A}}$ mice already manifested significant increase in BV/TV (30\%) and in cortical bone thickness (from 0.182 to $0.198 \mathrm{~mm}$ ) (Fig. 2A-C). In contrast, although an upward trend was observed in male Rage $^{+/-}$mice, they did not display significant increase in either parameter (Fig. 2B-C). However, 10-week old female Rage $^{+-}$mice displayed a significant increase in BV/TV (49\%) which was almost identical to the increase observed in female Rage ${ }^{\mathrm{AHA} /+}$ mice (51\% increase) (Fig. S2). Of note, while the BV/TV value was significantly different between $\operatorname{Rage}^{+/-}$and Rage $^{-/-}$mice in both male and female mice, it was not significantly different between Rage $e^{\mathrm{AHA} /+}$ and Rage $\mathrm{AHA} / \mathrm{AHA}$ mice. Our findings suggest that RAGE-AHA mutation led to a haploinsufficient bone phenotype, and that gene dosage of RAGE also plays a role in determining the bone phenotype, especially in female mice.

\section{Osteoclastogenesis is impaired in Rage ${ }^{\mathrm{AHA} / \mathrm{AHA}}$ mice}

Tartrate-resistant acid phosphatase (TRAP) staining of tibia sections showed the number of

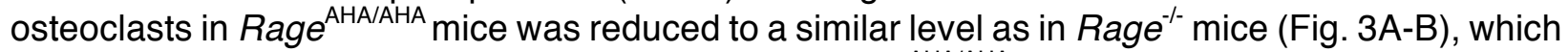
indicates a defect of osteoclast differentiation in Rage ${ }^{\mathrm{AHA} / \mathrm{AHA}}$ mice. To confirm this defect, we performed an osteoblast/bone marrow macrophage (BMM) co-culture osteoclastogenesis assay in vitro. In this model, WT primary calvarial osteoblasts were co-cultured with bone marrow macrophages (BMMs) from WT, Rage $\mathrm{AHA} / \mathrm{AHA}^{\mathrm{A}}$ and Rage $^{-/-}$mice to induce osteoclastogenesis from BMMs. Compared to the large and multinucleated TRAP-positive cells formed in cultures of WT BMMs, Rage $\mathrm{AHA} / \mathrm{AHA}$ and Rage ${ }^{-/-}$formed much smaller TRAP-positive cells (Fig. 3C). To better quantify the differences in osteoclast numbers among the three genotypes, we sub-grouped the osteoclasts based on the number of nuclei number they contain (Fig. 3D). Interestingly, while the number smaller osteoclasts (3-10 nuclei) were not significantly reduced in Rage $\mathrm{AHA} / \mathrm{AHA}^{\mathrm{A}}$ and Rage ${ }^{\prime-}$, the number of larger osteoclasts ( $>11$ nuclei) were substantially reduced (Fig. 3D). Collectively, these results demonstrate that impaired HS-RAGE interaction leads to abnormal maturation of osteoclasts, which suggests a key role for RAGE-HS interaction in regulating osteoclastic differentiation and function. 


\section{Rage $^{\text {AHA/AHA }}$ mice were protected from liver injury after APAP overdose}

We and others have previously reported that RAGE was responsible for neutrophil-mediated secondary injury (in an HMGB1-dependent manner) following acetaminophen (APAP)-induced liver necrosis $(3,6)$. When subjected to a sublethal dose of APAP, Rage ${ }^{-/-}$mice displayed a reduction of neutrophil infiltration and liver damage compared with WT mice. We thus similarly used this APAP overdose model to examine the role of HS-RAGE interaction in mediating neutrophilic liver injury. As expected, we found that like Rage ${ }^{-/-}$mice, Rage AHA/AHA $^{\text {mice were also }}$ protected from APAP-induced liver injury. The plasma level of alanine aminotransferase (ALT), a

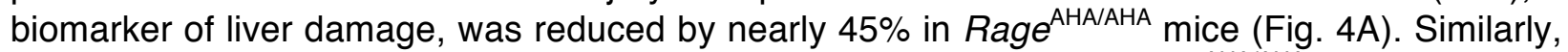

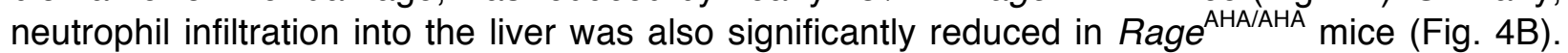
Histologic examination also confirmed that livers from Rage ${ }^{\mathrm{AHA} / \mathrm{AHA}}$ mice had significantly reduced necrotic area compared to the injured WT livers (Fig. 4C-D). Notably, these results were very similar to what we previously observed in Rage $^{-/}$mice (3). Our data strongly suggest that in this drug-induced liver injury model, HS-RAGE interaction plays an essential role in mediating HMGB1-dependent neutrophil infiltration.

\section{RNA-seq transcriptome analysis of neutrophils from Rage $^{\mathrm{AHA} / \mathrm{AHA}}$ mice and Rage $^{-/-}$mice}

To understand whether ablation of RAGE signaling has an impact on the general mobility of neutrophils, we performed an air pouch assay to examine LPS-induced neutrophil infiltration. Interestingly, in this assay, neutrophil infiltration was indistinguishable between WT, Rage $\mathrm{AHA} / \mathrm{AHA}$ and Rage $e^{-/-}$mice (Fig. 5A). This result confirmed that Rage $\mathrm{AHA} / \mathrm{AHA}$ and Rage ${ }^{-/-}$neutrophils have normal mobility when a different chemoattractant (other than HMGB1) was responsible for the infiltration. During this assay, we unexpectedly discovered that several common surface markers were dramatically upregulated on Rage $^{-/-}$neutrophils with $100 \%$ penetrance. Compared to WT neutrophils, the expression level of Ly6C and CD45 on Rage ${ }^{-/-}$neutrophils were 10-15 fold higher (Fig. 5B). Surprisingly, the expression levels of both markers were completely normal on

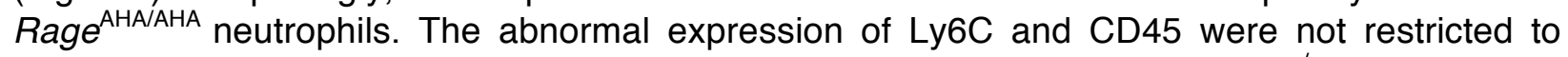
neutrophils as we also found they are overexpressed in T and B cells from Rage Ric $^{-/}$mice (Fig. S3). The fact that 2 out of 8 common leukocyte markers were highly elevated in Rage ${ }^{-/-}$(the other 6 markers were normal, including CD11b shown in Fig. 5B), but were normal in Rage AHA/AHA $^{-1}$ neutrophils strongly suggests that complete removal of RAGE protein has a much broader global impact compared to mere point mutations of RAGE. To grasp the full scale of the alteration in gene expression caused by knocking out Rage, we performed an RNA-seq analysis of mature neutrophils from WT, Rage ${ }^{\mathrm{AHA} / \mathrm{AHA}}$ and Rage $^{-/-}$mice.

Principle component analysis of the RAN-seq data found clear separation of transcriptomes of Rage ${ }^{\mathrm{AHA} / \mathrm{AHA}}$, Rage $\mathrm{R}^{-/-}$and WT mice, with all three biological replicates of each genotype closely clustering together (Fig. S4). As expected, the overall number of differently regulated genes (DEGs) in Rage ${ }^{-/-}$neutrophils were almost 2.5 times higher than in Rage AHA/AHA $^{\text {A }}$ neutrophils (603 vs. 247). Significant overlap was found in upregulated genes, with $80 \%$ of upregulated genes in Rage $\mathrm{AHA} / \mathrm{AHA}_{(143 / 178)}$ also being upregulated in Rage (-/- $^{-\mathrm{n}}$ neutrophils (Fig. 5C and Data file S1). In contrast, the overlap was rather limited in downregulated genes, with merely $29 \%(20 / 69)$ of downregulated genes in Rage ${ }^{\mathrm{AHA} / \mathrm{AHA}}$ also being downregulated in Rage ${ }^{-/-}$. Gene ontology analysis of upregulated genes found that genes related to regulation of leukocyte activation (43 genes), differentiation (27 genes), proliferation (29 genes), adhesion (34 genes) and regulation of cytokine production (30 genes) were highly enriched in Rage ${ }^{-/-}$neutrophils (Data file S2). The same biological processes were also enriched in Rage ${ }^{\mathrm{AHA} / \mathrm{AHA}}$ neutrophils, except that the number of genes involved were roughly half of the number of genes found in Rage ${ }^{-/-}$ neutrophils (Data file S3). These findings suggest that RAGE signaling plays a profound role in 
leukocyte biology. Gene ontology analysis of downregulated genes did not find any biological processes that are significantly differently regulated in either $\operatorname{Rage}^{-/-}$and $R a g e^{\mathrm{AHA} / \mathrm{AHA}}$ neutrophils. Curiously, we did identify several highly downregulated genes in Rage ${ }^{\mathrm{AHA} / \mathrm{AHA}}$ neutrophils that were

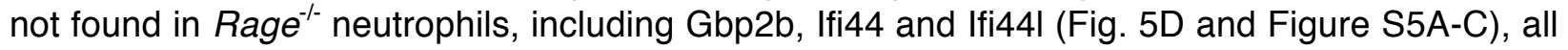
of which have been shown to be interferon $\gamma$-induced genes and play important roles in defense against infection (19-21). Many top DEGs in Rage ${ }^{-/-}$neutrophils were not found in Rage AHA/AHA $^{-1}$ neutrophils (Fig. 5E), which include highly upregulated genes : Rps3a2, Rps3a3, Rps13_ps1 and Rps15a_ps7, Tpm3_rs7 and Rnf5; and highly down regulated genes: Cxcl1, Muc1, Trim46, Ifi208 and BC147527. To our surprise, Ly6C and CD45 were not among the DEGs in Rage ${ }^{-/}$neutrophils, which suggests that either the posttranscriptional regulation or the secretion of Ly6C and CD45 were altered in Rage $^{-/-}$neutrophils. If that were the case, we would expect that the global protein expression profile of $R_{a g} e^{-/-}$neutrophils would perhaps be even more dramatically altered than what is apparent on RNA sequencing.

\section{RNA-seq transcriptome analysis of lung tissues from Rage $^{\mathrm{AHA} / \mathrm{AHA}}$ mice and Rage $^{-/-}$mice}

To gain further insight into the impact of impaired RAGE signaling on gene transcription, we performed RNA-seq analysis of lung tissue, an organ in which RAGE is abundantly expressed. Surprisingly, the number of DEGs were much less in lungs compared to neutrophils in both strains. We found 202 DEGs in Rage ${ }^{-/}$lungs and merely 31 DEGs in Rage AHA/AHA $_{\text {lungs (Fig. 5F }}$ and Data file S4). The difference between the two genotypes was however even more dramatic in lungs (7-fold) than we observed in neutrophils (2.5-fold). Similar to what we found in neutrophils, in Rage ${ }^{\mathrm{AHA} / \mathrm{AHA}}$ lung significant overlap was found in upregulated genes, with $50 \%$ of upregulated

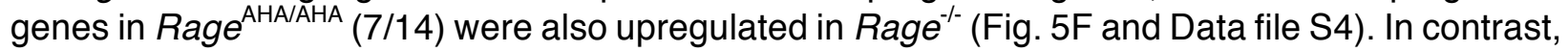
the overlap was very limited in downregulated genes, with merely $23.5 \%$ (4/17) of downregulated genes in Rage $e^{\mathrm{AHA} / \mathrm{AHA}}$ were similarly downregulated in Rage ${ }^{-/}$. Comparing the DEGs between lungs and neutrophils, we found that interestingly, many top DEGs appeared in both tissues in a genotype-specific manner. In Rage AHA/AHA, $\mathrm{Gbp} 2 \mathrm{~b}$, Ifi44 and Gm28438 were highly downregulated in both lungs and neutrophils (Fig. 5D and 5G, Figure S5D-E). In Rage ${ }^{-/}$, Rps3a2, Rps3a3, Rps13_ps1, Rps15a_ps7, Tpm3_rs7, Rf5, and BC147527 were differently regulated in both lungs and neutrophils to similar extent (Fig. 5E and H). Furthermore, two genes, Rpl34_ps1 and Gm42031, were found to be highly downregulated in both genotypes and in both tissues. As

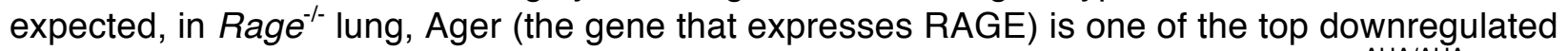

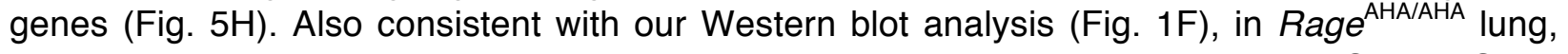
Ager was expressed at a similar level as in WT lung on RNA sequencing (Figure S5F). Gene ontology analysis revealed genes related to regulation of cell killing (11 genes), canonical Wnt signaling (7 genes) and epithelial cell proliferation (11 genes), among others, were highly enriched in Rage ${ }^{-/}$lung (Data file S5). In contrast, none of these biological processes were enriched in Rage ${ }^{\mathrm{AHA} / \mathrm{AHA}}$ lung due to limited number of DEGs.

\section{Development of a rabbit mAb that binds specifically to the HS-binding site of RAGE}

As Rage ${ }^{\mathrm{AHA} / \mathrm{AHA}}$ mice phenocopy Rage $^{-/}$mice in both osteoclastogenesis and response to APAPinduced liver injury, inhibition of HS-RAGE interaction could be an effective means to block RAGE signaling. To this end, we developed a rabbit monoclonal antibody (mAb B2) to specifically target HS-RAGE interaction. The epitope of B2 includes the HS-binding residues R216 and R218. Compared to the apparent binding affinity between B2 and WT RAGE $(0.1 \mathrm{nM})$, the affinity between B2 and R216A-R218A mutant was dramatically reduced to $4 \mathrm{nM}$ (Fig. 6A). Using a heparin Sepharose column, we confirmed that B2 was indeed able to significantly inhibit the binding of RAGE to heparin (Fig. 6B). Importantly, while a polyclonal anti-RAGE antibody significantly inhibited the binding between RAGE and its ligands HMGB1 and S100B, B2 did not 
interfere with the binding between RAGE and its ligands (Fig. 6C-D). The specificity of B2 was determined by immunostaining of lung sections from WT and Rage ${ }^{-/}$mice. RAGE specific staining was observed in WT lung section while no staining was shown on Rage ${ }^{-/-}$(Fig. 6E). Western-blot analysis of whole lung lysate from WT, Rage ${ }^{\mathrm{AHA} / \mathrm{AHA}}$ and $R a g e^{-/-}$mice further showed that B2 was highly specific for RAGE but failed to detect the RAGE-AHA mutant, which again confirmed the epitope of B2 includes R216 and R218 (Fig. 6F).

Using a human breast cancer MDA-MB-453 cell line, which was known to overexpress RAGE (22), we examined B2 binding to cell surface RAGE by FACS. As expected, B2 stains 453 cells extremely well, displayed a 2-log shift compared to IgG control (Fig. 6F). Interestingly, when 453 cells were pretreated with heparin lyase III (HL-III) to remove cell surface HS, binding of B2 IgG was dramatically reduced by 20 -fold (Fig. 6F). Because removal of HS would dissociate cell surface oligomeric RAGE back to monomers, we suspect that B2 preferably binds to dimeric RAGE in a bivalent fashion. Indeed, when Fab fragment of B2 was tested on 453 cells, we found that its cell surface binding was much weaker (due to monovalent interaction), and the binding showed minimal sensitivity to HL-III treatment.

\section{B2 inhibits RAGE-dependent biological processes in cell and animal models}

We first tested the inhibitory activity of B2 on RAGE-dependent osteoclastogenesis. BMMs was co-cultured with primary calvarial osteoblasts in the presence of $20 \mu \mathrm{g} / \mathrm{ml}$ of B2 or isotypematched control antibody. As expected, cells treated with B2 formed fewer and smaller TRAPpositive cells, especially for cells with more than 10 nuclei (Fig. 7A-B). Remarkably, the osteoclastogenic phenotype of WT BMM treated with B2 was essentially identical that of Rage ${ }^{\mathrm{AHA} / \mathrm{AHA}}$ BMMs (Fig. 3C-D). In monoculture osteoclastogenesis assay, where BMMs was induced with $\mathrm{m}-\mathrm{CSF}$ and RANKL, B2 was equally effective in inhibiting osteoclast formation (Fig. C). The hepatoprotective effects of B2 were also examined in an APAP-induced acute liver failure model. In this experiment, mice were pretreated with $B 2$ at $10 \mathrm{mg} / \mathrm{kg}$ before injection of sublethal dose of APAP. Compared to control lgG treated mice, B2 was able to reduce plasma ALT concentration by $40 \%$ and neutrophil infiltration by $50 \%$ (Fig. 7C-D). Again, the level of protection provided by B2 was highly similar to what was observed in Rage AHA/AHA mice (Fig. 4A-B). Collectively, these results validated our strategy of targeting the HS-binding site of RAGE as a means to inhibits RAGE signaling.

\section{DISCUSSION}

While our previous work has shown that HS is a potent inducer of RAGE oligomerization and that HS-RAGE interaction is essential for RAGE signaling in endothelial cells (16), the physiological significance of HS-induced RAGE oligomerization remains unexplored due to a lack of genetic models. To address this gap, we generated a Rage ${ }^{\text {AHA/AHA }}$ knock-in mice to specifically disrupt HS-RAGE interactions. As the HS-binding site and the ligand binding site of RAGE are spatially separated (16), we were able to create a RAGE variant (R216A-R217H-R218A) with normal ligand binding, but impaired HS-binding capacity (Fig. 1). This novel mouse model allowed us to dissect the specific contribution of HS-RAGE interaction to RAGE signaling in both physiological and pathological conditions for the first time.

Zhou et al. first reported that Rage ${ }^{-/-}$mice display enhanced bone mineral density due to impaired osteoclastogenesis (9). Examination of the bone morphometric indexes of Rage AHA/AHA $^{\text {A }}$ mice found that they also display an osteopetrotic bone phenotype in both trabecular and cortical bones, with a severity that was equivalent to Rage ${ }^{-/-}$mice (Fig. 2B). Natural history study of the bone phenotype found that for both strains, the bone was largely normal at 4 weeks old (Fig. 2D), suggesting the osteoclastogenesis during the bone modeling phase is not dependent on RAGE signaling. The osteopetrotic phenotype became full-fledged when mice reach 10 weeks old (for 
both sexes), and the phenotype remained when mice reach 4 months of age. It is interesting that while the bone phenotypes of Rage $\mathrm{AHA} / \mathrm{AHA}^{\mathrm{A}}$ and $\mathrm{Rage}^{-/-}$mice were indistinguishable at 10-weeks for both males and females, their phenotypes at 4-weeks and 4-months were somewhat different. It is unclear what factors contributed to this, but we suspect that the extensive transcriptomic changes experienced by Rage ${ }^{-/-}$mice (Fig. 5) might have modified the bone phenotype in an agedependent manner. The fact that RAGE signaling regulates bone density in mature bones only, and that it regulates both trabecular and cortical bone homeostasis suggests that RAGE would be an attractive therapeutic target for treating diseases involving abnormal bone remodeling.

The fact that the Rage $\mathrm{AHA} /+$ mice are haploinsufficient suggests that RAGE ${ }^{\mathrm{AHA}}$ mutant protein plays a dominant effect on wildtype RAGE. The dominant negative role of RAGE ${ }^{\text {AHA }}$ mutant can only be explained in the light of RAGE oligomerization. In heterozygous Rage $e^{\mathrm{AHA} /+}$ mice, the chance of assembling a functional dimer with intact HS-binding site would be merely $25 \%(50 \% \times 50 \%)$. This represents a $75 \%$ loss of functional RAGE signaling complex compared to WT mice, which apparently was sufficiently severe to impair normal osteoclastogenesis. In contrast, in Rage $^{+-}$mice, because all expressed RAGE are wildtype, they have $100 \%$ of chance to form functional RAGE signaling complex. The fact that male Rage $^{+/}$mice displayed slightly elevated bone density (although not significant) could be attributed to somewhat reduced overall expression level of RAGE, which can be reasonably expected from heterozygous mice. Interestingly, this gene dosage effect was in full play in 10 weeks old female mice, where the $\mathrm{Rage}^{+/-}$mice did show significantly increased bone volume (49\%), which was identical to the increase observed in Rage ${ }^{\mathrm{AHA} /+}$ mice (51\%). In summary, our analysis has shown for the first time that mice are highly sensitive to the quantity of functional RAGE oligomeric complex, and that female mice appeared to be more sensitive to this regulation than the males.

Our genetic manipulation of HS-RAGE interaction has provided clear evidence that disrupting HS-RAGE interaction pharmacologically would be an effective means to block RAGE signaling. To validate this novel concept, we developed an $m A b$ (B2) that specifically targets the HS-binding site of RAGE. The epitope of B2 includes R216 and R218, the exact residues that we mutated in Rage $\mathrm{AHA} / \mathrm{AHA}$ mice. Accordingly, B2 treatment of WT BMMs precisely copied the osteoclastogenesis phenotype of Rage $e^{\mathrm{AHA} / \mathrm{AHA}}$ BMMs (Fig. 7A-C and Fig. 3C-D). Similarly, B2 treatment also provided significant protection against APAP-induced liver injury, and the extent of protection in liver damage and suppression in neutrophil infiltration was highly similar to what was observed in Rage ${ }^{\mathrm{AHA} / \mathrm{AHA}}$ mice (Fig. 7D-E and Fig. 4). Combined, these data show that HS-RAGE interaction can be effectively targeted pharmacologically.

From a pharmacological point of view, targeting the HS-binding site of RAGE bears two main advantages over strategies targeting the ligand binding site of RAGE. First, targeting the HS-binding site of RAGE can in principle block the signaling of all RAGE ligands because HSdependent RAGE oligomerization is a common mechanism of RAGE signaling irrespective of the ligands involved. This is difficult to achieve for inhibitors that target the ligand binding site of RAGE because the binding sites for different RAGE ligands are not identical (18, 23-25). Secondly, inhibitors that target the HS-binding site of RAGE leave the ligand-binding site of RAGE unoccupied, which would be free to interact with its multiple ligands that are overexpressed in disease states. Such inhibitors may thus turn RAGE into a decoy receptor, which is able to soak up ligands without transducing signal. In principle, this would greatly lower the risk of undesirable side effects by preventing spillover of unbound RAGE ligands to other inflammatory receptors such as TLR4, with which RAGE share many ligands (26-29).

By comparing transcriptomes of neutrophils and lung tissues from Rage ${ }^{\mathrm{AHA} / \mathrm{AHA}}$ and Rage ${ }^{1-}$ mice, we present here clear evidence that complete deficiency of RAGE had much broader impact on global gene expression compared to point mutations of RAGE. Under the assumption that RAGE signaling is abolished in Rage ${ }^{\mathrm{AHA} / \mathrm{AHA}}$ mice, which was true in both animal models we 
tested, we predict that most of the additional DEGs found in Rage ${ }^{-/}$mice were perhaps not associated with RAGE signaling. Presumably, the expression levels of these genes were changed due to certain compensatory mechanisms for the sheer loss of RAGE protein. Because some of the changes in expression level were truly dramatic in both mRNA and protein level, it is highly plausible that some of these changes will manifest as phenotypes in Rage $e^{-/-}$mice in certain disease models, but in reality might have little to do with RAGE signaling. While it can be argued that the additional DEGs found in Rage $^{-/}$mice are due to HS-independent RAGE signaling, such position cannot be well defended for the following reason. If RAGE signaling really consists of

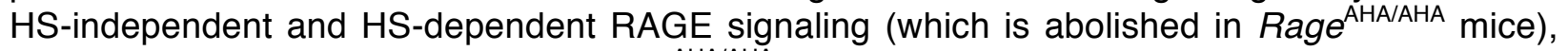

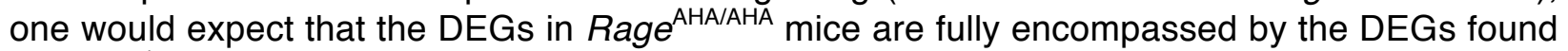
in Rage ${ }^{-/}$mice. However, this was not the case, especially for the downregulated genes (in both neutrophils and lungs, Fig. $5 \mathrm{C}$ and F), where many more downregulated genes are found uniquely in Rage AHA/AHA $^{\text {mice than the ones that overlaps with the downregulated genes in Rage }}$ ${ }^{1-}$ mice. Based on the scope of the inflammatory genes that are differently regulated in Rage ${ }^{-/-}$ neutrophils (and most likely in other leukocytes as well), we believe caution will need to be taken when using Rage ${ }^{-/-}$mice in inflammatory models. In many cases, the Rage ${ }^{\mathrm{AHA} / \mathrm{AHA}}$ mice might be a cleaner model for investigating the role of RAGE signaling.

The fact that most of the downregulated genes in Rage AHA/AHA $^{\text {mice are not found in Rage }}$ ${ }^{1-}$ mice is highly curious. Taking Gbp2b and Ifi44L as examples, the expression levels of both were

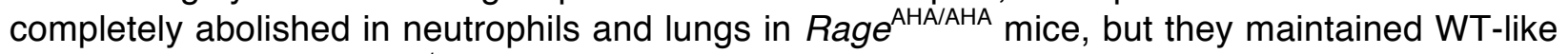
expression levels in Rage ${ }^{-/-}$mice (Figure S5). If Gbp2b and Ifi44L (both are interferon $\gamma$ responsive genes) were truly associated with RAGE activation, the only logical explanation would be that the activation of these genes was somehow preserved in Rage ${ }^{-/-}$mice. Taking into considerations that many RAGE ligands can also cross-activate TLR4, it is plausible that in the absence of RAGE, certain RAGE ligands activate TLR4 instead, resulting in induction of Gbp2b and Ifi44L. In contrast, because RAGE ${ }^{\text {AHA }}$ mutant behave essentially like a decoy receptor, being able to still bind the ligand without transducing signal, spillover of RAGE ligands to TLR4 would be unlikely. Indeed, LPS has been shown to be able to induce Gbp2b (synonymous with Gbp1) expression, which suggests that in theory activation of TLR4 with another ligand would also induce Gbp2b expression (20). Ongoing research in our lab seeks to establish the connection between RAGE signaling and Gbp2b-mediated defense signaling.

In summary, by using Rage $\mathrm{AHA} / \mathrm{AHA}$ mice, we presented strong evidence for an essential role of HS-RAGE interaction in bone remodeling and drug-induced liver injury. We further presented a new strategy in treating RAGE associated diseases by blocking the HS-binding site of RAGE using mAb. Based on our RNA-seq study, we expect that HS-RAGE interaction would plays essential roles in many inflammatory responses that involves other types of leukocytes. The Rage $\mathrm{AHA} / \mathrm{AHA}_{\text {mice }}$ and the anti-RAGE mAb antibody we reported here would certainly be invaluable tools to further our understanding of the pathophysiological roles of RAGE. To evaluate the therapeutic potential of $m A b B 2$, we plan to determine its efficacy in a number of inflammatory and tumor models and is in the process of humanization of B2 for testing in primates. A limitation of our study is that we only focused on a small portion of the HS-binding site located on C1 domain. It is perceivable that mAbs that target other epitopes within the HS-binding site would also effectively block RAGE signaling and we are hoping to identify more such mAbs to fully realize the therapeutic potential of RAGE antagonism. 


\section{MATERIALS AND METHODS}

\section{Generation of Rage $^{\mathrm{AHA} / \mathrm{AHA}}$ knock in mice}

The knock-in project was performed at the Gene Targeting and Transgenic Shared Resource of Roswell Park comprehensive cancer center. Briefly, the synthesized sgRNA (which targets Exon 6 of mouse rage gene as shown in Fig. 1), repair template ssODN and mRNA encodes for Cas9 were micro-injected into 1-cell stage fertilized egg of C57BL6J strain, which were then surgically transferred into pseudopregnant foster mothers also of C57BL6J background. Through CRIPSRbased homology-directed repair, the triple mutations were introduced into the rage locus. The pups were screened by Next Generation sequencing, and the mice that bear correct mutations were mated to WT C57BL6 mice to confirm germline transmission of the mutations. The F1 heterozygous mice were bred together to generate homozygous offspring. Rage ${ }^{-/}$mice were originally gifted by A. Bierhaus (University of Heidelberg, Heidelberg, Germany).

\section{MicroCT analysis}

Mouse femurs from different ages of WT, Rage $\mathrm{AHA} /+^{\mathrm{A}}, \operatorname{Rage}^{\mathrm{AHA} / \mathrm{AHA}}, \operatorname{Rage}^{+/-}$and Rage $^{-/-}$mice were harvested, fixed for 48 hours in 10\% neutral buffered formalin. A quantitative analysis of the gross bone morphology and microarchitecture was performed using ScanCo microCT 100 system (University at Buffalo). 3D reconstruction and bone microarchitecture analysis were performed using AnalyzePro (AnalyzeDirect Inc).

\section{TRAP staining}

Mouse tibia from 10-week old male mice were harvested, fixed for 48 hours in $10 \%$ neutral buffered formalin, and decalcified in 10\% EDTA for 2 weeks at RT. The samples were embedded in paraffin and sectioned at $5 \mu \mathrm{m}$ for TRAP staining. The sections were counter stained with Fast Green.

\section{Osteoclastogenesis Assay}

Primary osteoblasts were isolated from calvaria of 5-8-day old WT mice following an established protocol (30). Osteoblasts $\left(5 \times 10^{3}\right.$ cells/well) were seeded in a 96-well plate the day before starting the co-culture. Freshly isolated bone marrow cells (from WT, Rage $\mathrm{AHA} / \mathrm{AHA}^{\mathrm{A}}$ and Rage $\mathrm{R}^{-/-}$mice) were suspended in $10 \mathrm{ml}$ of a-MEM containing $10 \% \mathrm{FBS}$ and $1 \mathrm{X}$ penicillin/streptomycin, $10^{-7} \mathrm{M}$ dexamethasone, and $10^{-8} \mathrm{M} 1 \mathrm{a}$ - and 25-dihydroxyvitamin D3. $100 \mu \mathrm{l}$ of bone marrow cells were added into each well. The medium was replaced every 2 days thereafter until the appearance of giant osteoclasts. For B2 inhibition assay, $20 \mu \mathrm{g} / \mathrm{ml} \mathrm{B2-lgG}$ or control lgG was included in the medium in selected wells. To visualize osteoclasts, the cells were fixed and stained for TRAP activity using a Leukocyte Acid Phosphatase kit (Sigma). Osteoclasts were sub-grouped into four categories based on the number of nuclei and quantified to analyze the population of osteoclasts with different number of nuclei.

\section{Mouse model of APAP liver injury}

WT, Rage $\mathrm{AHA} / \mathrm{AHA}^{\mathrm{A}}$ and Rage $^{-/-}$mice were fasted overnight (12 to 15 hours) to deplete GSH stores before APAP (Sigma) administration. Fresh APAP was dissolved in warm $\left(\sim 50^{\circ} \mathrm{C}\right)$ sterile saline, cooled to $37^{\circ} \mathrm{C}$, and injected intraperitoneally at $300 \mathrm{mg} / \mathrm{kg}$. The plasma samples were collected 24 hours after APAP administrations for measuring ALT concentration. In general, the concentration of ALT reached its highest point at 24 hours after APAP overdose. For B2 protective treatment, WT mice were injected intraperitoneally with $10 \mathrm{mg} / \mathrm{kg}$ of B2-lgG $12 \mathrm{~h}$ before APAP overdose $(300 \mathrm{mg} / \mathrm{kg})$. Plasma ALT was measured using the ALT Infinity reagent (Thermo Fisher Scientific) following the manufacturer's instructions. Livers were harvested, fixed for 24 hours in 
$10 \%$ neutral buffered formalin. Samples were further embedded in paraffin and sectioned at $4 \mu \mathrm{m}$ for H\&E staining. The necrosis area was quantified with ImageJ.

\section{Neutrophil infiltration in liver necrosis}

The identical liver lobe from different mice were collected, minced and digested with $0.2 \%$ collagenase $A$ for 1 hour at 37 degree with shaking, and the digested tissue was further dissociated into single cell suspension by pipetting several times. Cells passed though 70 um cell strainer were pelleted, resuspended in $1 \mathrm{ml} \mathrm{PBS}$ and overlayed onto $4 \mathrm{ml}$ of $33 \%$ Percoll gradient. After centrifugation at RT for 15 min at $1400 \mathrm{~g}$, cell pellet was collected as leukocytes which include polymorphonuclear cells (PMN). Leukocytes were further treated with ACK Lysing Buffer for a short time to remove red blood cells and counted. The number of neutrophils were quantified by FACS after staining with rat anti-mouse Ly-6G-PE, Clone 1A8 (Biolegend), rat anti-mouse CD45FITC (Biolegend), and rat anti-mouse CD11b-APC (Biolegend) for 15 min at 4 degree. Ly-6G high cells were counted as neutrophils.

\section{Air pouch model and neutrophil (PMN) isolation}

$3 \mathrm{ml}$ of sterile air was injected under the dorsal skin of WT, Rage $e^{\mathrm{AHA} / \mathrm{AHA}}$ and Rage ${ }^{-/-}$mice. Three days later, the pouch was refilled with $2 \mathrm{ml}$ of sterile air. On the seventh day, $1 \mu \mathrm{g}$ of LPS from Escherichia coli O111:B4 (sigma) in $0.5 \%$ solution of carboxymethyl cellulose (sodium salt; Sigma) was injected into the air pouch. After 4 hours, mice were euthanized by isoflurane inhalation and the cells in the pouch were collected by washing with PBS. Cells were counted and stained with Ly-6G-PE to determine the percentage of PMNs.

\section{Leukocyte cell surface marker analysis}

For analysis of PMNs, total cells collected from the air pouch were stained with Ly-6G-PE, Clone $1 \mathrm{~A} 8$, rat anti-mouse CD45-FITC, and rat anti-mouse CD11b-APC, rat anti-mouse Ly-6C-PE, rat anti-mouse F4-80-PE for 15 min at 4 degree.

For analysis of $T$ and $B$ cells, spleenocytes were stained with rat anti-mouse CD45-FITC, rat antimouse Ly-6C-PE, rat anti-mouse F4-80-PE, rat anti-mouse CD3-PE, rat anti-mouse CD4-APC and rat anti-mouse CD8-APC. CD3+ cells were gated as T cells (including both CD4+ and CD8+ cells), and CD3- cells were gated as B cells.

\section{RNA-seq transcriptome analysis}

LPS-induced leucocytes (> 95\% PMNs) were collected from air pouch as described above and used for RNA extraction. For lung tissue samples, $30 \mathrm{mg}$ thoroughly perfused lungs were homogenized by bullet blender 5E (Next Advance). Total RNA was prepared using RNeasy Mini Kit (QIAGEN) following the protocol. cDNA library construction and RNA sequencing were performed at Novogene Co. Inc (Sacramento, CA). RNA integrity and quantitation were assessed using the RNA Nano 6000 Assay Kit of the Bioanalyzer 2100 system (Agilent Technologies, CA, USA). A total amount of $1 \mu \mathrm{g}$ RNA per sample was used as input material for the RNA sample preparations. Sequencing libraries were generated using NEBNext Ultra RNA Library Prep Kit for Illumina (NEB, USA) following manufacturer's recommendations. The clustering of the indexcoded samples was performed on a cBot Cluster Generation System using PE Cluster Kit cBotHS (Illumina) according to the manufacturer's instructions. After cluster generation, the library preparations were sequenced on an Illumina platform PE150 and paired-end reads (150bp) were generated. Reference genome and gene model annotation files were downloaded from genome website browser (NCBI/UCSC/Ensembl, mm10) directly. Paired-end clean reads were aligned to the reference genome using Spliced Transcripts Alignment to a Reference (STAR) (31). 
DESeq2 R package (v1.30.1) was used for gene count normalization and differential expression analysis(32). The list of Rage $\mathrm{AHA} / \mathrm{AHA}^{\mathrm{A}}$ and Rage ${ }^{-/-}$differentially expressed genes compared to WT was filtered by an absolute $\log _{2}$ fold change $\geq 1$ and a multiple testing corrected $\mathrm{p}$-value $\leq 0.05$. This list of differentially expressed genes in Rage ${ }^{\mathrm{AHA} / \mathrm{AHA}}$ and Rage ${ }^{-/}$lungs or PMNs were further analyzed and annotated with Gene Ontology (GO) analysis using clusterProfiler R package (v3.18.1) (33). A multiple testing corrected $p$-value $\leq 0.05$ cutoff was used for the applied conditional hypergeometric test for GO term overrepresentation of $\mathrm{GO}$ terms in molecular function and biological process ontologies. The used GO annotations were obtained from the Bioconductor Mus musculus annotation package. PMN and lung RNA-sequencing data have been deposited into the NCBI Gene Expression Omnibus database (accession number GSE174178).

\section{Development of B2}

Rabbit monoclonal antibodies (mAbs) were raised against the murine RAGE V-C1 domain in its native conformation. The rabbit hybridomas were generated at Epitomics Inc. (now part of Abcam). The hybridoma line $240 \mathrm{E}-\mathrm{W} 3$ was used to fuse with rabbit spleenocytes. Out of the 4,000 hybridoma clones that were screened, around 100 were able to detect mVC1 by direct ELISA. The supernatants of the positive clones were then screened for mAbs that specifically interacted with the HS-binding site. In this screening, instead of using WT mVC1, various mVC1 mutants (K43A-K44A, K39A-R104A, K107A, and R216A-R218A) were immobilized to ELISA plates. These mutants bear alanine mutations in the HS-binding residues. In this assay, mAbs were sought that show reduced binding to the mutants compared to the WT mVC1. In principle, the reduced binding would only occur if the residues that we mutated belong to the epitope recognized by the mAb. In this assay the epitopes of B2 was found to include R216 and R218. Rabbit B2 was purified from hybridoma supernatant by Protein A chromatography.

\section{Direct ELISA for screening RAGE-binding mAbs}

200 ng of human RAGE extracellular domain or R216A-R218A mutant were immobilized onto 96well high-binding ELISA plate. Plates were blocked with 5\% BSA in PBS and incubated with 0.01 to $10 \mathrm{nM}$ of rabbit $\mathrm{B} 2 \mathrm{mAb}$ for 1 hour at room temperature. Bound $\mathrm{B} 2 \mathrm{mAbs}$ were quantitated with anti-rabbit-HRP (Jackson Immunology) followed by the addition of HRP substrate (Thermo Scientific).

\section{Recombinant expression of B2}

To produce B2 mAbs in large quantity for in vivo studies, we cloned the variable regions of B2 from hybridoma cells and grafted them onto human IgG1 constant region. The chimeric antibody was expressed in Chinese Hamster Ovary (CHO) cells. The pcDNA5_FRT plasmid purchased from Invitrogen was modified to insert the variable regions of $B 2$ heavy and light chains in human IgG1 constant regions. Then the plasmid was transfected into $\mathrm{CHO}$ _Flp cells using lipofectamine ${ }^{\circledR} 3000$ Reagent (Invitrogen), and the positive clones will be screened out over ten days in the presence of Hygromycin B $(50 \mathrm{mg} / \mathrm{mL}$, Gibco). Cells with positive expression of the antibody were grown in CD CHO AGT expression medium (ThermoFisher) with supplemental glutamine, D+ glucose, and Pen/Strep for two weeks. The media was then harvested, and the secreted antibody (IHH-lgG) was purified using a $5 \mathrm{~mL}$ HiTrap protein $\mathrm{G}$ column (Cytiva). The antibodies were buffer exchanged into PBS, and the concentration was determined using NanoDrop Spectrophotometer (ThermoFisher). The purity of each batch was examined using SDS-PAGE. Purified antibodies were stored at $4^{\circ} \mathrm{C}$ before experiments. For B2-Fab, human chimeric B2 were digested with immobilized papain using Pierce ${ }^{\mathrm{TM}}$ Fab Preparation kit (ThermoFisher) following the instructions. 


\section{Effect of B2 on RAGE binding on heparin}

$30 \mu \mathrm{g}$ of mouse mVC1 were either directly loaded onto heparin Sepharose column, or loaded after 30 mins incubation with $68 \mu \mathrm{g}$ Fab fragment of B2 (1:1 molar ratio). B2-Fab bound mVC1 displayed greatly reduced binding to heparin column. The bound $\mathrm{mVC1}$ was eluted with a salt gradient from $200 \mathrm{mM}$ to $1.4 \mathrm{M} \mathrm{NaCl}, \mathrm{pH} 7.1$ in HEPES buffer.

\section{B2 cell surface binding by FACS}

Breast tumor MDA-453 were lifted from culture dish using Accutase (Biolegend) and incubated with human B2-IgG or B2-Fab in $100 \mu \mathrm{L}$ PBS and $0.1 \%$ bovine serum albumin for $45 \mathrm{~min}$ at $4{ }^{\circ} \mathrm{C}$. Bound B2-IgG was stained with goat anti-human IgG-Alexa 568 (1:1,000; Invitrogen) for 30 min and analyzed by FACS. B2-Fab was stained with biotinylated goat anti-human IgG $(1: 1,000$, Invitrogen) followed by streptavidin-PE staining. In some experiments, cells were pretreated with recombinant heparin lyases III (5 milliunits $/ \mathrm{ml}$ ) for $15 \mathrm{~min}$ at room temperature prior to binding experiments.

\section{Immunohistochemistry}

Lungs from WT and Rage ${ }^{-/}$mice were perfused, harvested, and fixed for 24 hours in $10 \%$ neutral buffered formalin. Samples were further embedded in paraffin and sectioned at $5 \mu \mathrm{m}$ for RAGE staining with $1 \mu \mathrm{g} / \mathrm{ml}$ human B2-lgG. After staining with biotinylated rabbit anti-human IgG secondary antibody (1:200), the sections were developed using the ABC system (Vector Laboratories) and the cell nuclei were counter stained with $15 \%$ Ehrlich's hema- toxylin (Electron Microscopy Sciences).

\section{Effect of antibodies on RAGE-ligand binding}

HMGB1 or S100b (200 ng) were immobilized and the plate was blocked with $5 \%$ BSA. Biotinylated-mouse B2 V-C1 domain $(200 \mathrm{ng} / \mathrm{ml})$ was pre-incubated with rabbit lgG, polyclonal or monoclonal anti-RAGE (all at $5 \mu \mathrm{g} / \mathrm{ml}$ ) for 30 minutes at room temperature before being added to the plate. The percentage of antibody inhibition was calculated by comparing the absorbance obtained in the presence of specific antibodies to the absorbance obtained in the presence of control rabbit IgG (which was set to $100 \%$ ).

Expression of recombinant murine and human RAGE. Wildtype mouse RAGE V-C1 domains (mVC1-WT) and human SRAGE (V-C1-C2 domains) were produced in E.coli cells. Purification was carried out using HiTrap SP cation exchange column at $\mathrm{pH} 7.8$, followed by SEC on a Superdex 200 column (GE healthcare). The recombinant protein was purified to $>98 \%$ pure as examined by silver staining. Mouse RAGE triple mutant R216A-R217H-R218A (mVC1-AHA) was confirmed by sequencing and purified as described above for WT protein.

Heparin-Sepharose Chromatography and analytical Size-exclusion Chromatography. To characterize the binding of $\mathrm{mVC} 1$ and mutant to heparin, $100 \mu \mathrm{g}$ of purified $\mathrm{mVC} 1-\mathrm{WT}$ or $\mathrm{mVC} 1-$ AHA were applied to a HiTrap heparin-Sepharose column and eluted with a salt gradient from $200 \mathrm{mM}$ to $1.4 \mathrm{M} \mathrm{NaCl}, \mathrm{pH} 7.1$ (HEPES buffer). The conductivity measurements at the peak of the elution were converted to the concentration of $\mathrm{NaCl}$ based on a standard curve. For analyses of mVC1 and heparan sulfate dodecasaccharide (H12) complex, purified mVC1-WT or mVC1AHA $(40 \mu \mathrm{g})$ were incubated with HS dodecasaccharide $(\mathrm{H} 12,4 \mu \mathrm{g})$ in $20 \mathrm{mM}$ Tris, $150 \mathrm{mM} \mathrm{NaCl}$, $\mathrm{pH} 7.4$, at $4{ }^{\circ} \mathrm{C}$ over night. All complexes were resolved on a Superdex $200(10 / 300 \mathrm{~mm}) \mathrm{gel}$ filtration column using $20 \mathrm{mM}$ Tris, $150 \mathrm{mM} \mathrm{NaCl}$, $\mathrm{pH} 7.4$, at $4{ }^{\circ} \mathrm{C}$. 
HMGB1 binding ELISA. Binding affinity of mRAGE-WT and mRAGE-AHA to HMGB1 was measured by ELISA. Briefly, 96-well plate was coated by 200ng of mRAGE-WT and mRAGEAHA protein and blocked by $1 \%$ BSA. Biotinylated HMGB1 with concentrations from 50 ng to $1000 \mathrm{ng} / \mathrm{ml}$ were added into wells and incubated for 2 hours followed by incubation with streptavidin-HRP for $30 \mathrm{~min}$. $50 \mu$ l of HRP substrate solution was added for developing, and the reaction was stopped by adding $50 \mu \mathrm{l}$ of $1 \mathrm{M} \mathrm{H}_{2} \mathrm{SO}_{4}$. The absorbance at $450 \mathrm{~nm}$ was measured by a plate reader. Apparent $K_{d}$ value was calculated using Prism software.

Western blot analysis. One lung lobe from WT, Rage $\mathrm{AHA} / \mathrm{AHA}^{\mathrm{A}}$ or Rage ${ }^{-/-}$mice was collected and homogenized/lysed in lysis buffer $(50 \mathrm{mM}$ Tris- $\mathrm{HCl}, \mathrm{pH} 7.4,1 \%$ Triton $\mathrm{X}-100)$. After clearing by centrifugation, the lysate was boiled in SDS sample with $5 \% \beta$-mercaptoethanol and analyzed by gel electrophoresis using SureGel 4-20\% Bis-Tris gel (Genscripts). After transfer, the PVDF membrane was probed with a rat anti-RAGE mAb (R\&D system), rabbit anti-HMGB1 mAb (Abcam), or our rabbit B2.

\section{Statistical analysis}

All data are expressed as means \pm SEM. Statistical significance of differences between experimental and control groups was analyzed by two-tailed unpaired Student's test, between multiple groups by one-way analysis of variance (ANOVA) followed by Dunnett's or Tukey's multiple comparisons test using Prism software (version 7.03; GraphPad Software Inc.).

\section{AUTHOR CONTRIBUTIONS:}

Design research studies: ML, DKS, EPS, DX

Conduct experiments: ML, CO, LT, AV, DX

Acquire data: ML, CO, CJLA, LT, AV, YY, XZ, DX

Analyze data: ML, CO, CJLA, LT, AV, YY, XZ, DX

Write the manuscript: ML, CJLA, DKS, EPS, DX

\section{ACKNOWLEDGMENTS}

We thank the Optical Imaging and Analysis Facility of School of Dental Medicine, University at Buffalo, for assistance with $\mu \mathrm{CT}$ analysis. We also thank the Gene Targeting and Transgenic Shared Resource of Roswell Park Comprehensive Cancer Center for help with generating RAGE knock-in mice. This work is supported by National Institutes of Health grant R01AR07017 and R01HL094463 (to DX); R01GM125095 (to EPS); R01GM114179, R21Al138195 and R01CA246785 (to DKS); and Buffalo Accelerator Funds (to DX).

\section{Competing interests}

ML and DX are inventors for an international patent (pending, WO 2021/087462) that covers the sequence and use of anti-RAGE mAb B2. 


\section{REFERENCE}

1. B. I. Hudson, M. E. Lippman, Targeting RAGE Signaling in Inflammatory Disease. Annu. Rev. Med. 69, 349-364 (2018).

2. S. F. Yan, R. Ramasamy, A. M. Schmidt, The RAGE axis: a fundamental mechanism signaling danger to the vulnerable vasculature. Circ. Res. 106, 842-853 (2010).

3. K. Arnold et al., Design of anti-inflammatory heparan sulfate to protect against acetaminophen-induced acute liver failure. Sci. Transl. Med. 12 (2020).

4. E. Harja et al., Vascular and inflammatory stresses mediate atherosclerosis via RAGE and its ligands in apoE-/- mice. The Journal of clinical investigation 118, 183-194 (2008).

5. M. A. Hofmann et al., RAGE mediates a novel proinflammatory axis: a central cell surface receptor for S100/calgranulin polypeptides. Cell 97, 889-901 (1999).

6. P. Huebener et al., The HMGB1/RAGE axis triggers neutrophil-mediated injury amplification following necrosis. The Journal of clinical investigation 125, 539-550 (2015).

7. L. Park et al., Suppression of accelerated diabetic atherosclerosis by the soluble receptor for advanced glycation endproducts. Nature medicine 4, 1025-1031 (1998).

8. A. Taguchi et al., Blockade of RAGE-amphoterin signalling suppresses tumour growth and metastases. Nature 405, 354-360 (2000).

9. Z. Zhou et al., Regulation of osteoclast function and bone mass by RAGE. The Journal of experimental medicine 203, 1067-1080 (2006).

10. Z. Zhou et al., HMGB1 regulates RANKL-induced osteoclastogenesis in a manner dependent on RAGE. Journal of bone and mineral research : the official journal of the American Society for Bone and Mineral Research 23, 1084-1096 (2008).

11. J. Xie et al., Structural basis for pattern recognition by the receptor for advanced glycation end products (RAGE). J. Biol. Chem. 283, 27255-27269 (2008).

12. I. Popa, E. Ganea, S. M. Petrescu, Expression and subcellular localization of RAGE in melanoma cells. Biochem. Cell Biol. 92, 127-136 (2014).

13. H. Zong et al., Homodimerization is essential for the receptor for advanced glycation end products (RAGE)-mediated signal transduction. J. Biol. Chem. 285, 23137-23146 (2010).

14. A. Moysa, D. Hammerschmid, R. H. Szczepanowski, F. Sobott, M. Dadlez, Enhanced oligomerization of full-length RAGE by synergy of the interaction of its domains. Sci. Rep. 9, 20332 (2019).

15. J. Xue et al., Change in the Molecular Dimension of a RAGE-Ligand Complex Triggers RAGE Signaling. Structure 24, 1509-1522 (2016).

16. D. Xu et al., Stable RAGE-heparan sulfate complexes are essential for signal transduction. ACS Chem. Biol. 8, 1611-1620 (2013).

17. B. M. Dattilo et al., The extracellular region of the receptor for advanced glycation end products is composed of two independent structural units. Biochemistry 46, 6957-6970 (2007).

18. M. Koch et al., Structural basis for ligand recognition and activation of RAGE. Structure 18, 1342-1352 (2010).

19. J. C. Santos, P. Broz, Sensing of invading pathogens by GBPs: At the crossroads between cell-autonomous and innate immunity. J. Leukoc. Biol. 104, 729-735 (2018).

20. B. H. Kim et al., A family of IFN-gamma-inducible 65-kD GTPases protects against bacterial infection. Science 332, 717-721 (2011).

21. D. C. Busse et al., Interferon-Induced Protein 44 and Interferon-Induced Protein 44-Like Restrict Replication of Respiratory Syncytial Virus. J. Virol. 94 (2020).

22. M. W. Nasser et al., RAGE mediates S100A7-induced breast cancer growth and metastasis by modulating the tumor microenvironment. Cancer research 75, 974-985 (2015).

23. J. Xue et al., Advanced glycation end product recognition by the receptor for AGEs. Structure 19, 722-732 (2011).

24. T. Ostendorp et al., Structural and functional insights into RAGE activation by multimeric S100B. EMBO J 26, 3868-3878 (2007).

25. E. Leclerc, G. Fritz, M. Weibel, C. W. Heizmann, A. Galichet, S100B and S100A6 differentially modulate cell survival by interacting with distinct RAGE (receptor for advanced glycation end products) immunoglobulin domains. J. Biol. Chem. 282, 31317-31331 (2007).

26. H. Yang et al., A critical cysteine is required for HMGB1 binding to Toll-like receptor 4 and activation of macrophage cytokine release. Proc. Natl. Acad. Sci. U. S. A. 107, 11942-11947 (2010). 
27. T. Vogl et al., Mrp8 and Mrp14 are endogenous activators of Toll-like receptor 4, promoting lethal, endotoxin-induced shock. Nature medicine 13, 1042-1049 (2007).

28. D. Foell et al., Proinflammatory S100A12 can activate human monocytes via Toll-like receptor 4. Am. J. Respir. Crit. Care Med. 187, 1324-1334 (2013).

29. G. Esposito et al., Palmitoylethanolamide improves colon inflammation through an enteric glia/toll like receptor 4-dependent PPAR-alpha activation. Gut 63, 1300-1312 (2014).

30. S. E. Taylor, M. Shah, I. R. Orriss, Generation of rodent and human osteoblasts. Bonekey Rep 3, 585 (2014).

31. A. Dobin et al., STAR: ultrafast universal RNA-seq aligner. Bioinformatics 29, 15-21 (2013).

32. M. I. Love, W. Huber, S. Anders, Moderated estimation of fold change and dispersion for RNA-seq data with DESeq2. Genome Biol. 15, 550 (2014).

33. G. Yu, L. G. Wang, Y. Han, Q. Y. He, clusterProfiler: an R package for comparing biological themes among gene clusters. OMICS 16, 284-287 (2012). 


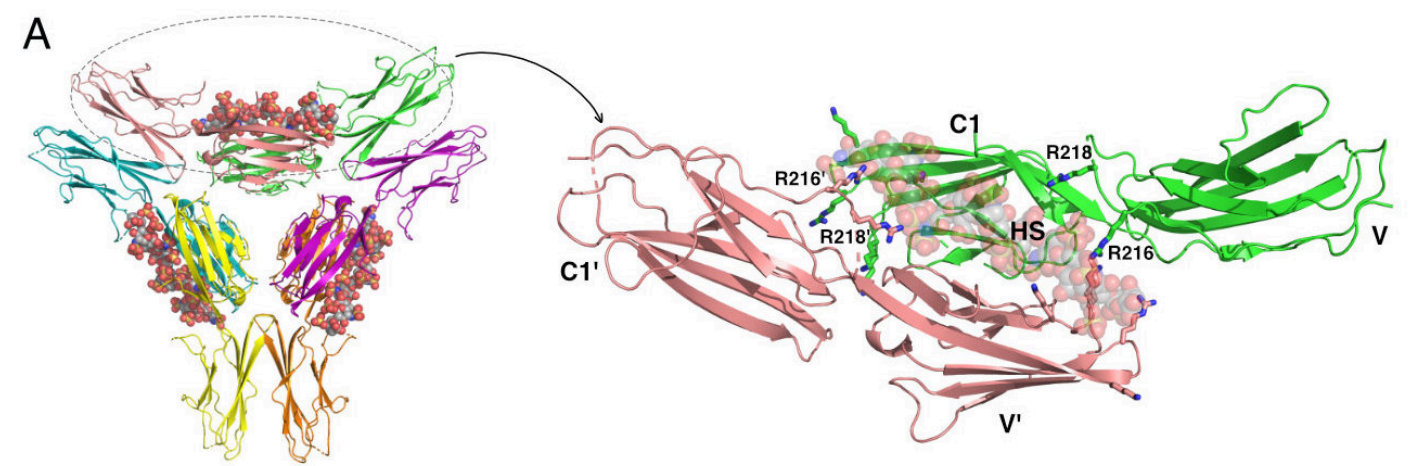

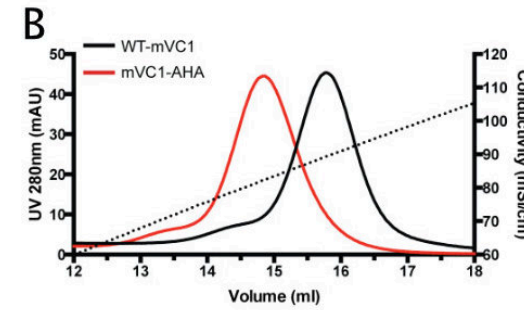

C
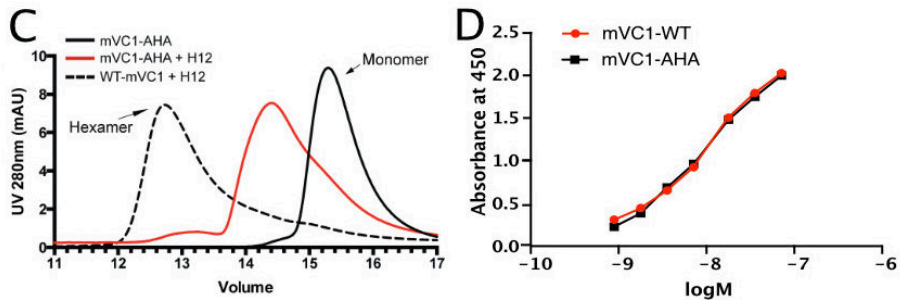

$\mathrm{E}$
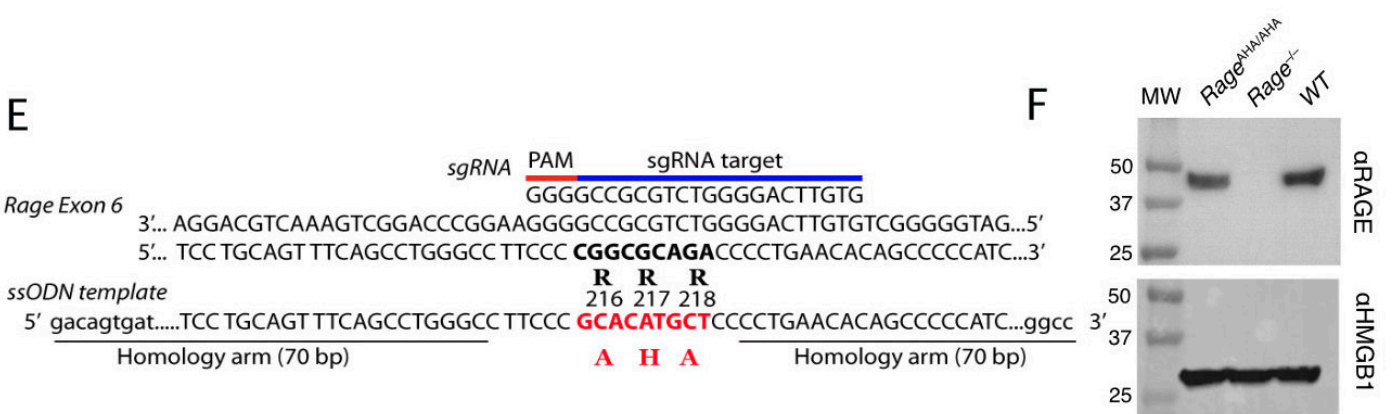

Figure 1 Characterization of $\mathrm{HS}$ binding-deficient RAGE mutant $\left(R^{216} R^{217} R^{218}\right.$ to $\left.A^{216} H^{217} A^{218}\right)$ and

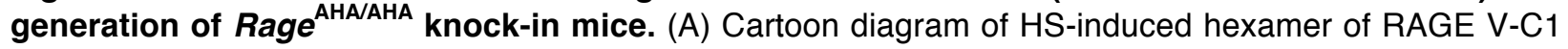
domains (PDB 4IMB). The hexamer is organized as trimer of dimers, with each dimer stabilized by one molecule of HS oligosaccharide. Oligosaccharides are shown in space-filling models. One dimer (salmon and green) is enlarged to show HS binding residues (in stick representation). R216 and R218 from both monomers (V-C1 and V'-C1') are marked. (B) Binding of wild-type murine RAGE V-C1 domain (mVC1WT) and RAGE-AHA mutant (mVC1-AHA) to heparin Sepharose column. RAGE-AHA mutant had reduced HS-binding capacity. (C) mVC1-WT or mVC1-AHA were incubated with HS dodecasaccharide (H12) and the mixtures were resolved on a Superdex $200(10 / 300 \mathrm{~mm})$ gel filtration column. H12 was unable to induce RAGE-AHA to form a stable RAGE hexamer. (D) Binding affinity of mVC1-WT and mVC1-AHA to immobilized HMGB1 was determined by enzyme-linked immunosorbent assay (ELISA). RAGE-AHA displayed WT-like binding affinity to ligand. (E) Targeting strategy for generating Rage AHA/AHA knock-in mice. Sequences of the targeting single guide RNA (sgRNA), the mutation sites in Rage exon 6, and the repairing template single-stranded donor oligonucleotides (ssODN) are shown. ( $F$ ) Western blotting analysis of RAGE expression in lung lysate. Top panel, lung lysates from WT, Rage AHA/AHA , Rage ${ }^{-/-}$mice were blotted with a rat anti-RAGE mAb (R\&D system), which showed RAGE was expressed at normal level in Rage AHA/AHA $^{\mathrm{A} i c e .}$ Bottom panel, as a protein loading control, the blot was reprobed with anti-HMGB1, which is universally expressed by all cells. 
A

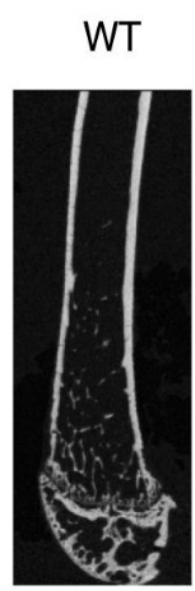

B
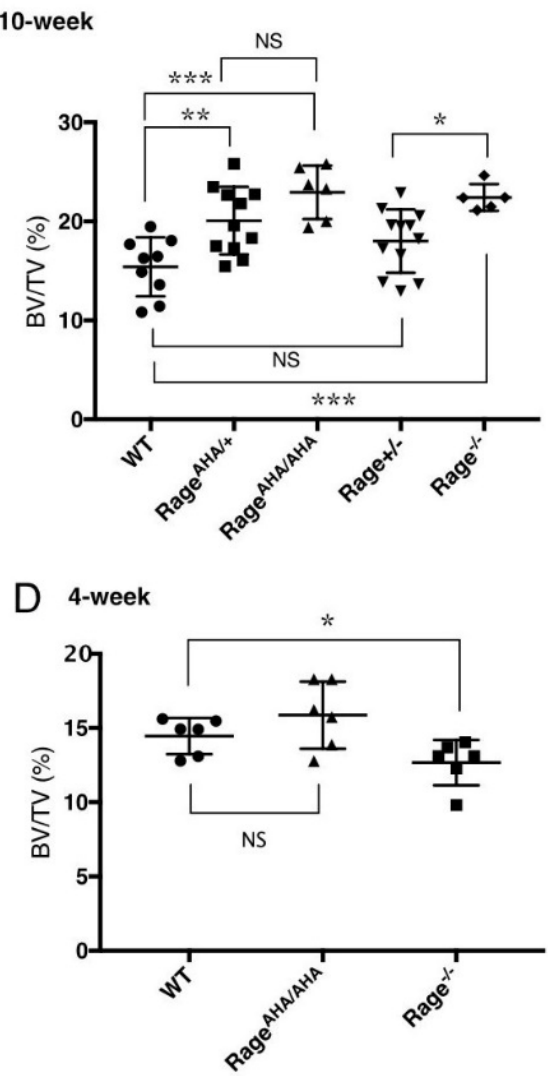
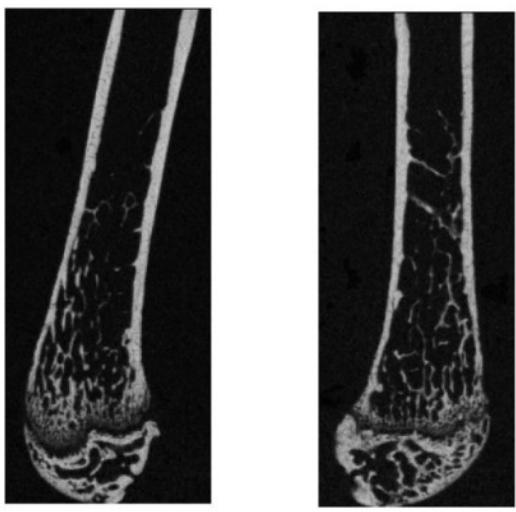

C

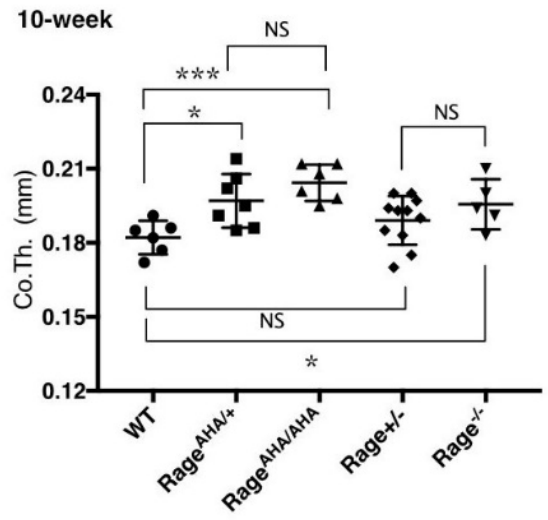

$\mathrm{E}$

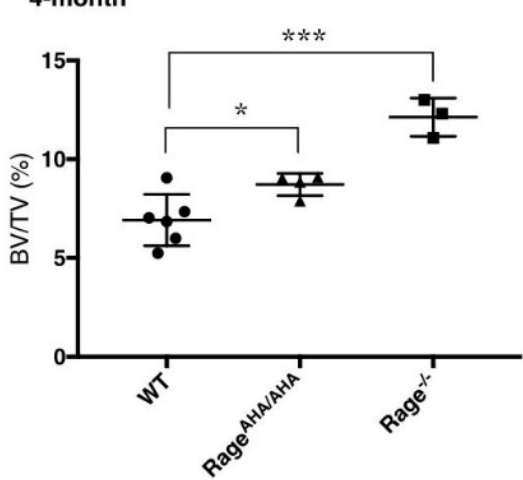

Figure 2. Rage $e^{\mathrm{AHA} / \mathrm{AHA}}$ and $R a g e^{\mathrm{AHA} / \mathrm{t}}$ mice develop osteopetrotic phenotype. (A) Representative $\mu \mathrm{CT}$ images of the femurs from 10-week old male WT, Rage ${ }^{\mathrm{AHA} / \mathrm{AHA}}$, Rage $\mathrm{AHA} /+^{\mathrm{A}}$ and Rage $e^{-/-}$mice. (B) Trabecular bone volume / tissue volume ratio (BV/TV), $n=5-12$, and (C) cortical bone thickness of femurs from 10week old male WT, Rage $e^{\mathrm{AHA} / \mathrm{AHA}}$, Rage $\mathrm{AHA} /+^{\text {, }}$, Rage ${ }^{+/-}$and Rage ${ }^{-/-}$mice, $\mathrm{n}=5-12$. (D) Trabecular BV/TV of 4week old male WT, Rage ${ }^{\mathrm{AHA} / \mathrm{AHA}}$ and Rage $e^{-/-}$mice, $\mathrm{n}=6$. (E) Trabecular BV/TV of 4-month old male WT, $\operatorname{Rage}^{\mathrm{AHA} / \mathrm{AHA}}$ and Rage ${ }^{-l-}$ mice, $\mathrm{n}=3-6$. Error bars represent $\mathrm{SD} .{ }^{*},{ }^{* *}$ and ${ }^{* * *}$ represents $\mathrm{P}<0.05,0.01$ and 0.001 respectively. 


\section{A}
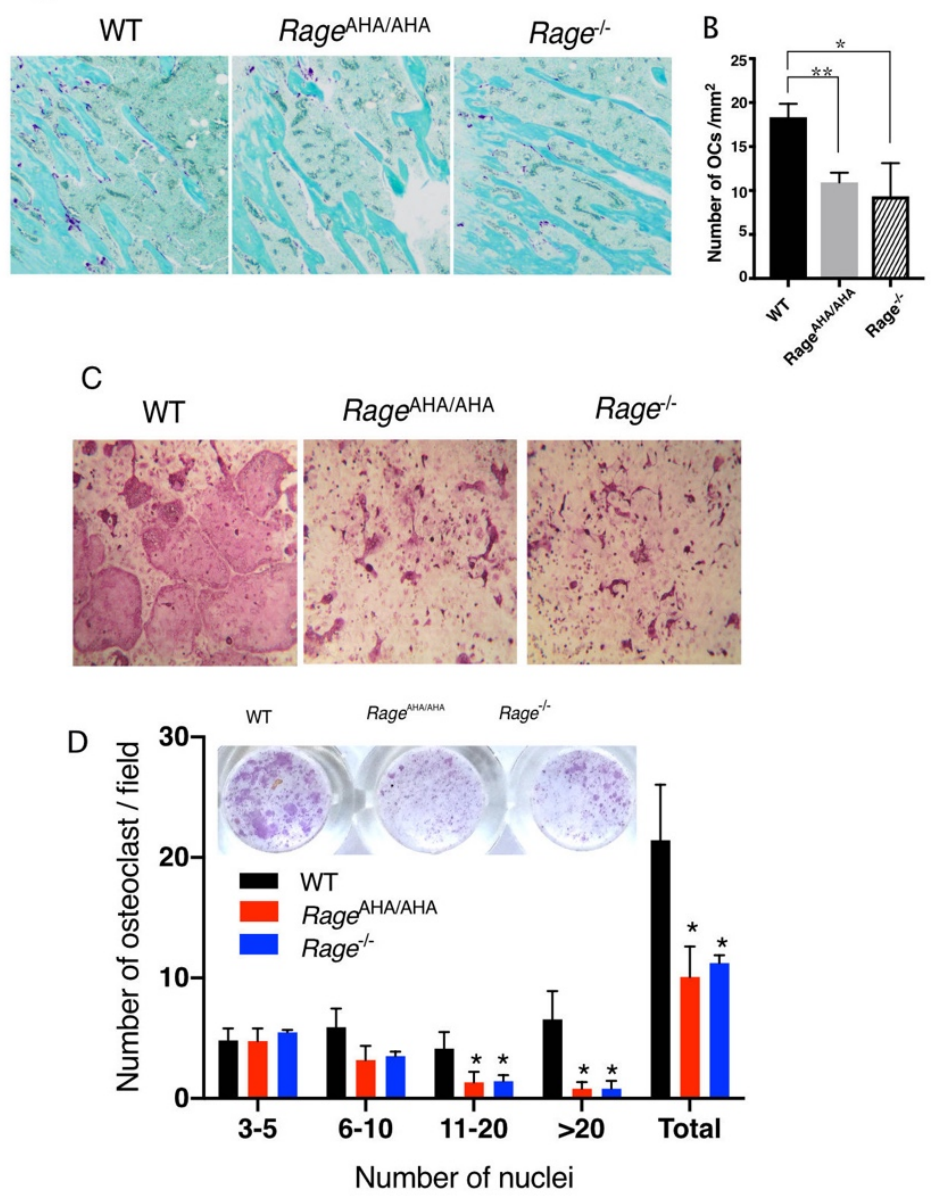

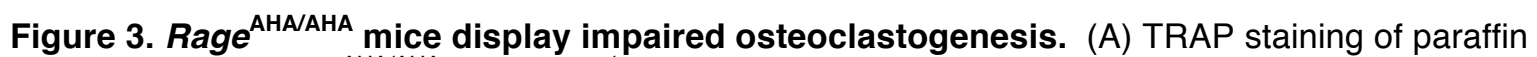
sections of WT, Rage $\mathrm{AHA} / \mathrm{AHA}^{\mathrm{A}}$ and Rage ${ }^{-/-}$tibias. Mature osteoclasts were stained purple, and bone was counterstained with Fast Green. (B) Quantification of osteoclasts (OCs) in tibia sections. $n=3$ mice. (C) BMM isolated from WT, Rage $e^{\mathrm{AHA} / \mathrm{AHA}}$, or Rage ${ }^{-/-}$mice were co-cultured with WT osteoblasts. Mature osteoclasts are visualized by TRAP staining. (D) Quantification of the number of osteoclasts per 100x field. Osteoclasts were sub-grouped into four categories based on the number of nuclei they contain. $\mathrm{n}=$ 3 wells. ${ }^{*}$ and ${ }^{* *}$ represents $\mathrm{P}<0.05$ and 0.01 respectively. Data are representative of at least three separate assays. 
A

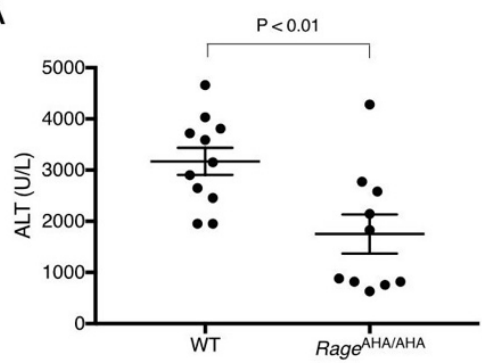

B

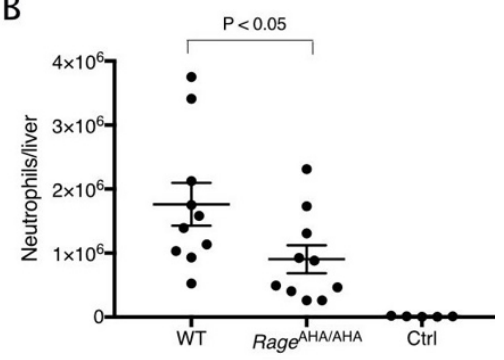

C

WT

$\operatorname{Rage}^{\mathrm{AHA} / \mathrm{AHA}}$
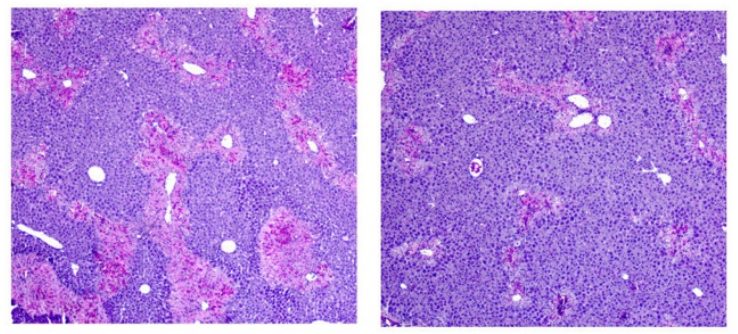

D

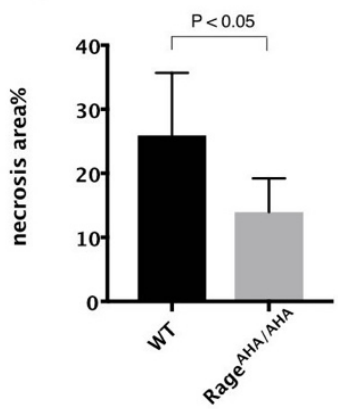

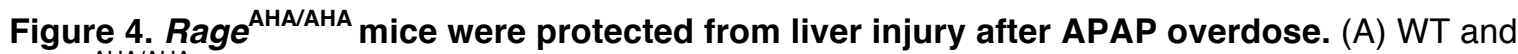

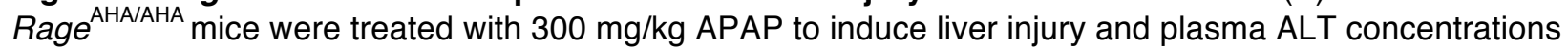
were measure 24 hours post injury, $n=10-11$. (B) Neutrophils recruitment into to the liver after APAPinduced liver injury, $n=5-10$. (C) Hematoxylin and eosin (H\&E) staining of paraffin-embedded liver tissues shown the extent of liver necrosis. (D) The necrotic area of H\&E stained liver tissues was quantified with Image $\mathrm{J}, \mathrm{n}=5$. 


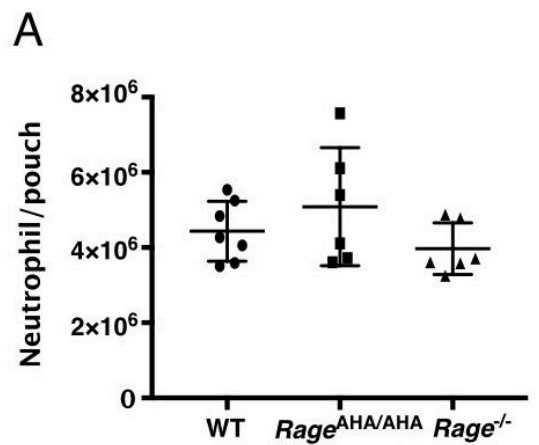

B
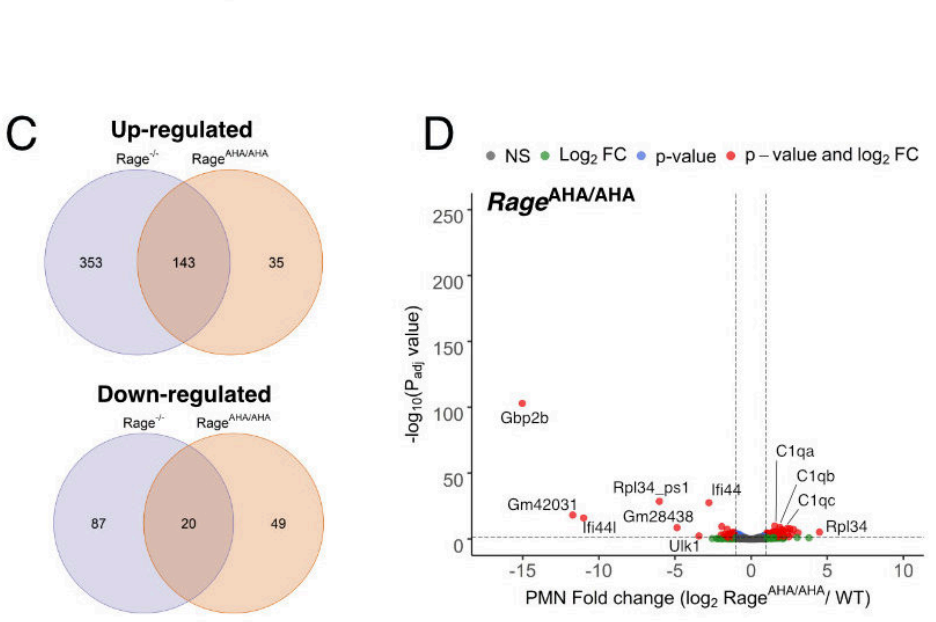

E $\cdot N S \cdot \log _{2} F C \cdot p$-value $\bullet p$-value and $\log _{2} F C$
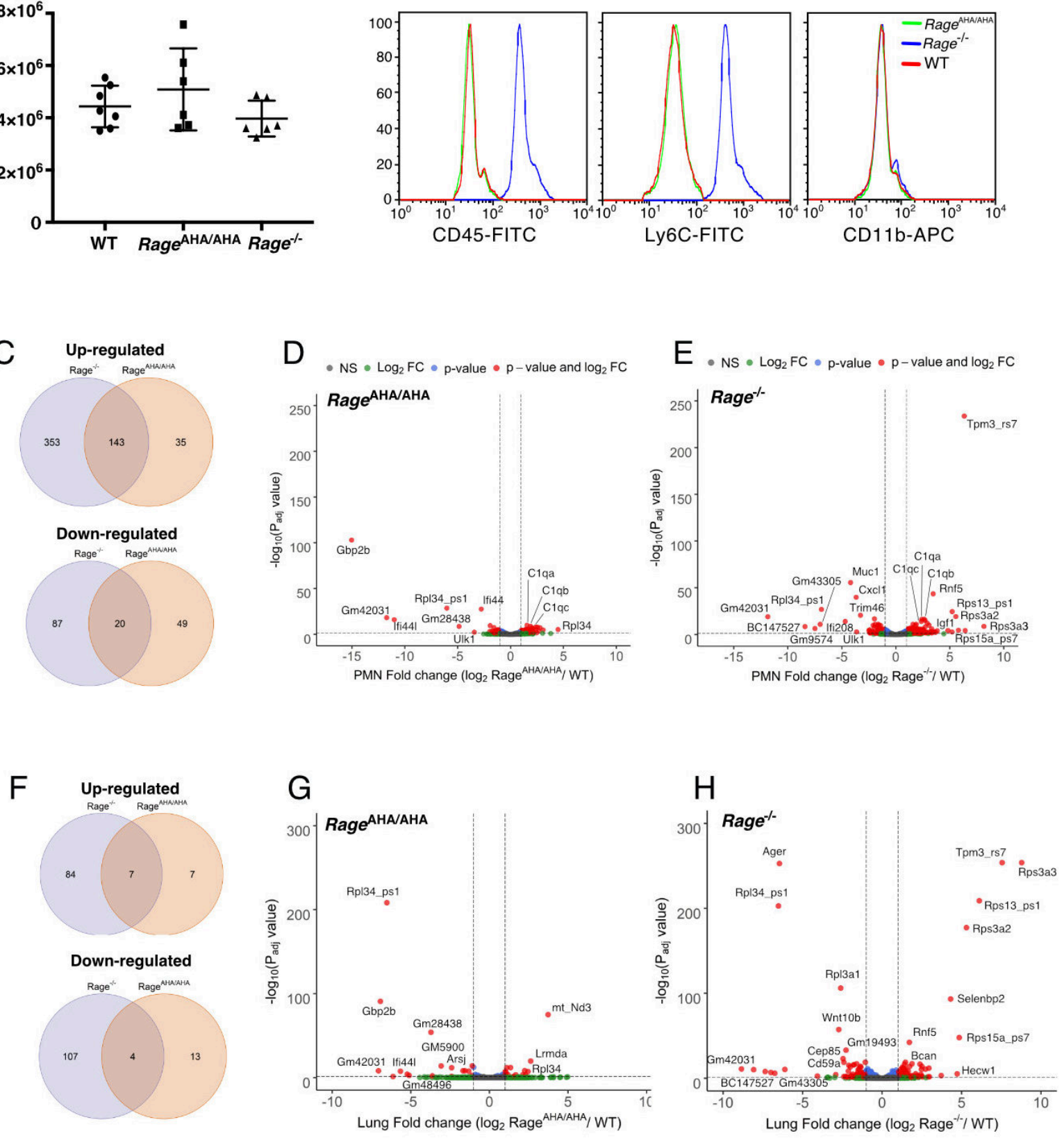

Figure 5. RNA-seq transcriptome analysis of $\operatorname{Rage}^{\mathrm{AHA} / \mathrm{AHA}}$ mice and Rage $\boldsymbol{e}^{-/-}$mice. (A) LPS-induced neutrophil infiltration into air pouch in WT, Rage ${ }^{\text {AHA/AHA }}$ and $R_{a g} e^{-/-}$mice, $n=6-7$. Neutrophils were quantified 4 hours after LPS injection. (B) Surface marker (CD45, Ly6C and CD11b) analysis of air pouch neutrophils by FACS. Cells from 6 mice of each genotype were analyzed with identical result. (C-E) RNA-seq transcriptome analysis of Rage ${ }^{\text {AHA/AHA }}$ and Rage ${ }^{-/-}$mice PMN. (C) Plot of up- and down-regulated genes in Rage $^{\text {AHA/AHA }}$ and Rage ${ }^{-/-}$PMN. DEGs between Rage ${ }^{\text {AHA/AHA }}$ and WT PMNs were determined based on Benjamin-Hochberg multicomparison correction p-values. 178 genes were found to be significantly upregulated $\left(p_{\text {adj }}\right.$ value $\leq 0.05, \log _{2}$ fold change $\geq 1$ ) while 69 genes were found to be significantly downregulated $\left(p_{\text {adj }}\right.$ value $\leq 0.05, \log _{2}$ fold change $\left.\leq-1\right)$. DEGs between Rage ${ }^{-/-}$and WT PMNs were determined similaly. 476 genes were found to be significantly up-regulated ( $p_{\text {adj }}$ value $\leq 0.05, \log _{2}$ fold change $\geq 1$ ) while 
107 genes were found to be significantly down-regulated $\left(p_{\text {adj }}\right.$ value $\leq 0.05, \log _{2}$ fold change $\left.\leq-1\right)$. (D) Volcano plot of DEGs between Rage ${ }^{\text {AHA/AHA }}$ PMN and WT PMNs. Genes with most significant chances were marked in the plot. (E) Volcano plot of DEGs between Rage ${ }^{-/-}$PMN and WT PMNs. (F-H) RNA-seq transcriptome analysis of whole lungs from Rage ${ }^{\mathrm{AHA} / \mathrm{AHA}}$ and Rage ${ }^{-/-}$mice. $(\mathrm{F})$ Plot of up- and down-regulated genes in Rage ${ }^{A H A / A H A}$ and Rage ${ }^{-/-}$lungs. 14 genes were found to be significantly up-regulated while 17 genes were found to be significantly down-regulated in Rage ${ }^{\mathrm{AHA} / \mathrm{AHA}}$ lungs. In Rage $\mathrm{e}^{-/-}$lungs, 91 genes were found to be significantly up-regulated while 111 genes were found to be significantly down-regulated. $(G)$ Volcano plot of DEGs between Rage ${ }^{\mathrm{AH} / \mathrm{AHA}}$ and WT lungs. Genes with most significant chances were marked in the plot. $(\mathrm{H})$ Volcano plot of DEGs between Rage ${ }^{-/-}$and WT lungs. 
A

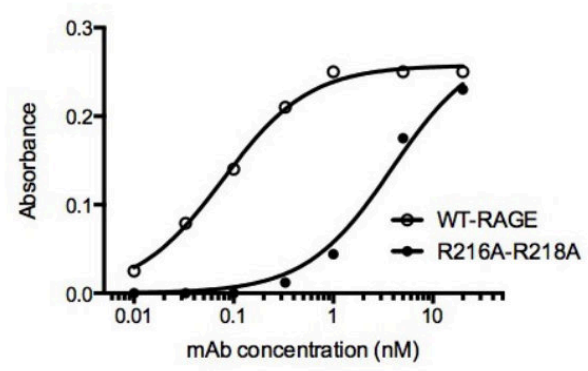

B

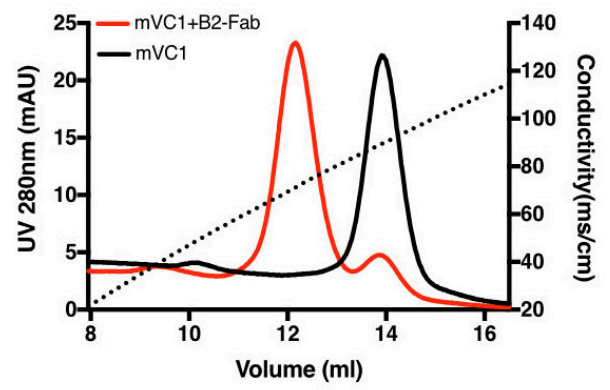

C

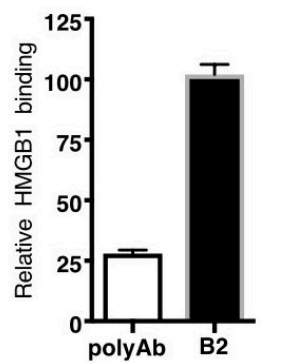

D

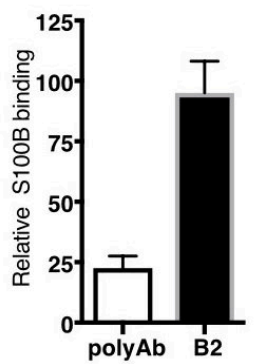

$\mathrm{E}$

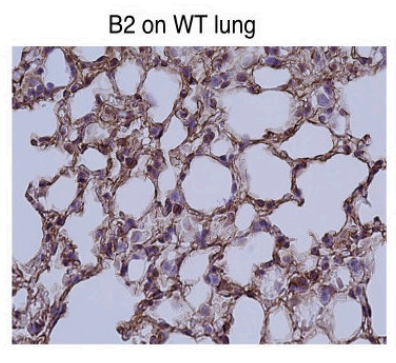

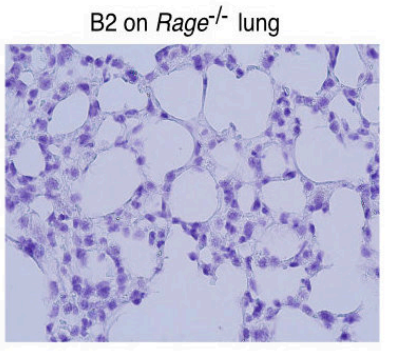

$\mathrm{H}$

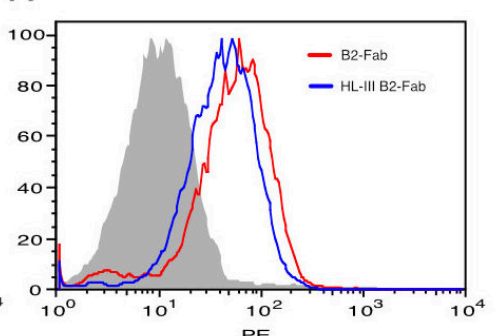

Figure 6. Development of a rabbit mAb that targets specifically to the HS-binding site of RAGE. (A) Binding of rabbit mAb B2 to immobilized wild-type sRAGE or R216A-R218A mutant was determined by ELISA. The dramatically reduced binding affinity of B2 to R216A-R218A mutant ( $\left.K_{d}=4 \mathrm{nM}\right)$ compared to its affinity to WT sRAGE $\left(K_{\mathrm{d}}=0.1 \mathrm{nM}\right)$ indicates that R216 and R218 are part of the epitope for B2. (B) B2 directly inhibits binding of RAGE to heparin. Wild-type mouse RAGE VC1 domain (mVC1) were either directly loaded onto heparin Sepharose column, or loaded after 30 mins incubation with Fab fragment of $\mathrm{B} 2$ at 1:1 molar ratio. B2-Fab bound $\mathrm{mVC} 1$ displayed greatly reduced binding to heparin column. (C) Binding of biotinylated SRAGE to immobilized HMGB1 was measured in the presence of anti-RAGE rabbit polyclonal $\mathrm{Ab}$ or $\mathrm{B} 2$ at $5 \mu \mathrm{g} / \mathrm{ml}$. Binding of sRAGE to HMGB1 in the absence of antibodies were set to $100 \%$. (D) Binding of biotinylated SRAGE to immobilized S100B was measured in the presence of antiRAGE rabbit polyclonal Ab or B2 at $5 \mu \mathrm{g} / \mathrm{ml}$. (E) Determine the specificity of B2 by immunostaining of lung sections from WT and Rage ${ }^{-/-}$mice with $1 \mu \mathrm{g} / \mathrm{ml}$ B2. (F) Western blot analysis of the specificity of B2 using lung lysate from WT, Rage $\mathrm{AHA}^{\mathrm{AHA}}$ and Rage $^{-/-}$mice. Please note B2 failed to detect RAGE-AHA mutant, again confirming the epitope of B2. (G) Binding of B2 IgG to untreated MDA-453 cells or cells pretreated with heparin lyase III (HL-III). Whole IgG form of B2 (bivalent) binds to cell surface RAGE in an HS-dependent manner. $(\mathrm{H})$ Binding of $B 2$ Fab fragment to untreated MDA-453 cells or cells pretreated with HL-III. Binding of the Fab form of B2 (monovalent) to RAGE was not sensitive to HL-III treatment. The shaded histograms in $(\mathrm{G}-\mathrm{H})$ are from cells stained only with control antibodies. 

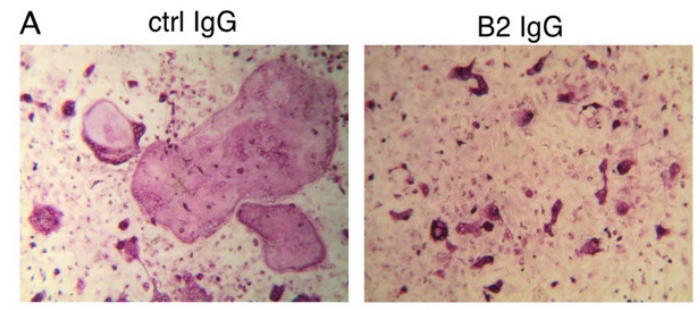

B

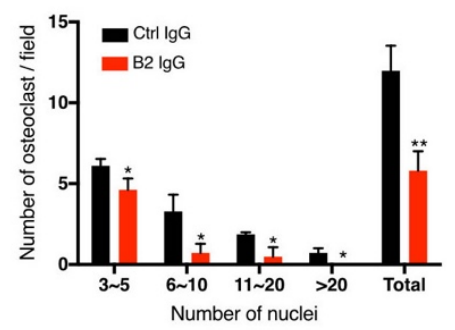

D

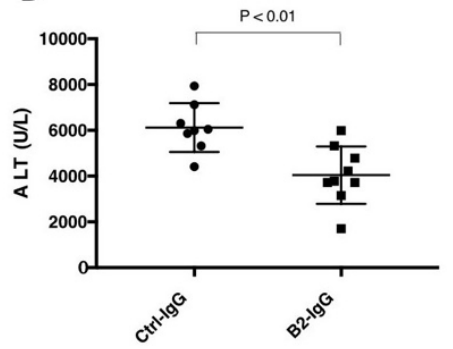

C

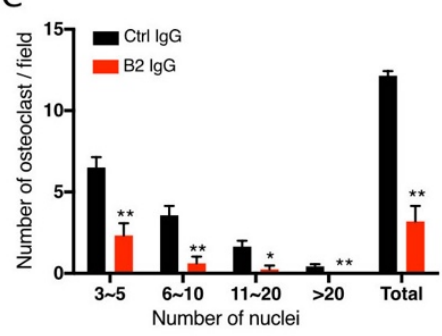

$\mathrm{E}$

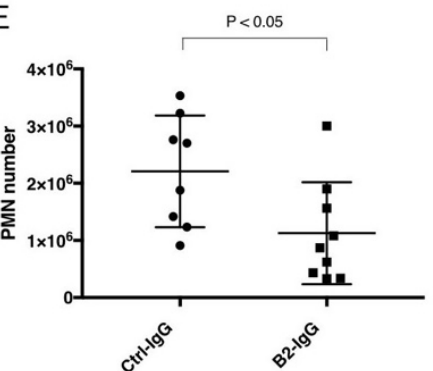

Figure 7. B2 inhibits RAGE-dependent biological processes in cell and animal models. (A) B2 inhibits osteoclastogenesis in vitro. Representative images of TRAP staining of WT bone marrow cells cocultured with WT osteoblasts in the presence of $20 \mu \mathrm{g} / \mathrm{ml} \mathrm{B2}$ or control IgG. Mature osteoclasts were stained pink. (B) Osteoclasts were sub-grouped into four categories based on the number of nuclei and quantified, $n=3$ wells. (C) B2 also inhibits osteoclastogenesis in monoculture assay, where differentiation of bone marrow macrophages was induced by exogenous RANKL and $m-C S F, n=3$ wells. (D-E) B2 was protective as a pretreatment in APAP-induced liver injury. WT mice were pretreated with $10 \mathrm{mg} / \mathrm{kg}$ of B2$\operatorname{lgG} 12 \mathrm{~h}$ before APAP overdose (300mg/kg). Plasma ALT concentrations (D) and neutrophils recruitment into to liver, $n=8-9(E)$ were quantified 24 hours post liver injury, $n=8-9$. 